\title{
Exploration: Ways and Functions of Oblivion
}

Oblivion is a knowledge problem. Whenever one becomes aware of having forgotten something, one knows that one does not know something. This is not ignorance, as the forgotten knowledge is knowledge that must have existed. Thus, oblivion is concerned with not-knowing-anymore; it is a special kind of not-knowing. ${ }^{1}$

Although it is not possible to unfold the long tradition of considering knowledge here, it still seems helpful to look at some basic elements of the concept of knowledge if the kinds and motifs of the loss of knowledge are supposed to be analysed. ${ }^{2}$ Knowledge, we may generally state, is the basis of all action. This general and - after all - culture-specific statement postulates that there is no movement within or into the world that is not based on a prerequisite from the past. No matter how good the movement's chance of being reflected by movers or observers is, it must be possible to place the change of a state of the world into a number of preceding changes.

According to the understanding of the occidental world, the concept of knowledge refers to the possibility of establishing relations of before and after, interdependencies and causal relations as well as causalities. If an observer succeeds with describing the change of a state of the world in a way that is plausible for others, this knowledge is considered true. The concept of knowledge aims at a category of observation or attribution; the entity which causes the movement does not require any talent for observation. Accordingly, thunder does not need to know that it follows lightning. This context demonstrates that any observation is a category of perception. The assumption that thunder comes after lightning results from an everyday observation which turns out to be wrong if the different speed of light and sound is considered.

Knowledge is considered a precondition for working on the world, no matter how much reflected on or assessed it is, after all. The conquest and control of nature may be successful due to making purposeful or programmatic use of

1 Peter Wehling (2006) carried out a comprehensive analysis of the concept of "not-knowing".

2 By reaching back to philosophical-knowledge-sociological general knowledge, the following elaborations are subject to the phenomenon of cryptomnesia which will be discussed in more detail elsewhere. 
knowledge. Thus, it is unimportant if the rain worm moves through the ground successfully and "skilfully" or if the physicist carries out a series of experiments at a particle accelerator. Likewise, it is unimportant if these two beings know what they know in the course of their actions. What is essential is the experience: the effect on the world. The - indeed culture-specific - habit of attributing knowledge to an "organism" rather than any other imaginable object seems much more significant.

From the activator's perspective, knowledge may be declarative or nondeclarative, explicit or implicit, conscious or unconscious. These three distinctions, taken from the concept of knowledge, are based on a common motif: communication and endowment with meaning-making. Whereas terming or attributing knowledge is always connected to meaning, there is a difference if such an attribution happens in the context of self-observation or if it does not. Meaning is a category of reason and thus of sense which, after all, comes from referring to things past. Meaning is thus closely connected to memory. ${ }^{3}$ If knowledge is a resource for changing states of the world, then meaning is a communicative selection with the purpose of understanding. ${ }^{4}$ In this context, it is an observer provided with knowledge, who carries out the meaningattributing selection; he or she does so at a particular social place within space and time. Thus, any working-on-the world becomes knowledge only by being combined with meaning attributed by oneself or by others; it may be strategic, explicit, implicit, intentional or instrumental knowledge or intuitive, implicit, habitual, routine or practical knowledge. ${ }^{5}$ Meaning would thus be a behaviouror action-related way of explicating knowledge. However, the reference to knowledge as a behavioural disposition or action-orientation is not arbitrary but depends on both situation and path. This means that certain perceived events address a particular experience in each case. When being thrown into the water, one starts making swimming movements - as far as one has learned how to do so - and does not start walking or riding a bicycle. This selectivity is an indication that not every kind of knowledge can be obtained at any

3 Thomas Khurana (2007) analyses the connection between the concepts of meaning and of memory from a systems-theoretical point of view. Gerd Sebald (2014) has presented a short social-phenomenological treatise on this issue.

4 According to Niklas Luhmann the theory of social systems deals first of all with systems of meaning.

5 Once again, here it becomes obvious that knowledge can only be understood to be a category of attribution - it is not about truth but at best about believing-to-be-true or about veridicality from the points of view of the observers involved. Accordingly, Peter L. Berger and Thomas Luckmann (1967, p. 26) keep the definition open, by understanding knowledge to be "that passes for knowledge in society". 
moment. Thus, what appears as knowledge is also much dependent on the context.

It is crucial, however, that knowledge is in principle constituted as experience based on past experiences. Individual experiences become experience only as far as they have left traces - the neuroscientists describe this as a change of the brain's synapse structure. The difference between individual experience and experience in general is that the concept of general experience refers to knowledge in the sense of imprints or traces which stay and may - explicitly or implicitly - affect behaviour or action. ${ }^{6}$ Thus, general experience refers to those elements of past events that the "organism" has stored. As soon as this storing may be expected, which is the case in social contexts, the problem of oblivion may occur. Thus, oblivion is not so much an actual loss of knowledge - because then the saying "what I do not know will not hurt me" makes sense - but rather a lack of specific knowledge assumed by a (self-)observer.

After these preliminary theoretical considerations, both measuring the range of the concept of oblivion and thoroughly analysing its sociological relevance by connecting to considerations on the concept of the social memory, it makes sense to look at some time-theoretical motifs. On this basis, it is possible to derive some basic principles about the remembrance of an "organism" referencing things past.

\subsection{Excursus: Time and Oblivion}

The concepts used so far for approaching oblivion have most of all been those of "knowledge" as well as the, up to here rather reluctantly addressed, "relatives" of the oblivion concept: memory and remembrance. This reluctance can be explained by the necessity of a closer analysis of the directions or interactions of memory, remembrance and oblivion. At first, however, it seems to be necessary to go far afield once again. When looking at how the terminology of memory is used, strikingly, the context within which the term is used, is usually not considered at the beginning of scientific reflection on storing knowledge or on retentivity. However, this is done when it is about the issue of the (social) constitution and construction of time. In other words: social-scientific considerations on issues of memory, remembrance and indeed oblivion begin

6 Experience piles up in the course of experienced events. The experience context consists of schemes of experience, in the context of which some of these experiences can no longer be explicated and serve as habitual basic preconditions for understanding the world (Schütz, 1967). 
with addressing time. Shedding light on this connection is the purpose of the following considerations, starting with some philosophies of time which have much influenced sociological thought. Also, it makes sense to look for aspects of addressing oblivion. In a second step, genuinely sociological theories of time will be outlined in the context of which another possible way of grounding the issue of memory becomes evident. This recently practised way addresses remembrance and oblivion and connects to works in cultural studies.

It is remarkable that in the interdisciplinary memory studies debate, the issue of "time" is hardly dealt with, not even among those sociologists dealing with memory. On the one hand, this blind spot may occur because research has not yet achieved the "depth" needed for discussing such a presuppositional and abstract concept. On the other hand, it may also be because research has considered issues of memory split and moved away from the issue of social time already at an early stage. No matter which reason for neglecting "time" we might prefer, some questions remain unanswered, especially when it comes to sociological theories.

Regardless of whether time is understood as an a priori category or as a cognitive-symbolic aid, it makes sense to approach the rarely explicated memory nature of the social with the help of the concept of time. In other words: it seems as if time as a concept of investigating the problem of consistency is more common than the concept of memory. Furthermore, such an approach seems to be necessary to work out another connection point to the phenomenon of oblivion: "Time heals".

Early evidence of considering time is found in ancient philosophy, from Aristotle to Plotinus as far as to Augustine, who, apart from a memory theory, developed an elaborated concept of time in his Confessiones. ${ }^{7}$ On the one hand, frequently used ways of structuring time are separating before from after; on the other hand, they create the tripartition of the continuum of time into past, present and future. Additionally, there is the distinction of an "objective" natural time - which is e. g. concluded from the movement of the stars - from subjectively perceived time. First of all, the sociological analysis is interested in those kinds of time starting from consciousness and the concluding consequences for the measurement of time or for regimes of time. After all, even an "objective" world time could only be concluded from subjective consciousness. Finally, another approach of considering time is delimiting it from its relation to space and is discussing this relation.

One fundamental problem of philosophising on time is the insight that time cannot be experienced as time - we cannot watch time passing, and also killing

7 On this see in more detail Johann Kreuzer (1995). 
time or passing time have not much to do with any analytical concept of time. This becomes particularly obvious if we try to experience the present, for any moment we would like to grasp consciously will be over the next moment. Time is fluent, and the present cannot be "experienced as itself within itself" but always only later. As soon as we deal with the present, we can only do so in the mode of a remembered present (Lotz, 2001, p. 659). The fluidity of the present results in revaluating the past or - given subjective consciousness - memory. However, suppose present is just resentfulness or making something present. In that case, it always includes an aspect of absence because the remembered event which once was present can only be symbolically doubled - as a memorised image, as a linguistic symbol or as a narration - but never reconstructed as the original. Simply because of the difference between the original experience and the memory of this experience and the memory of the memory resulting from repeating the process, a "feeling" of time can develop. It does derive from memory, but it is based on the perception of a difference resulting from the difference between an original experience, its theoretical and always symbolic duplication, and the reduplication of this duplication.

\subsubsection{Time and Inner Continuity}

At the beginning of the 2oth century, Henri Bergson's works on time and memory gained much popularity. ${ }^{8}$ However, soon the Bergson enthusiasm in the sciences faded away, which can be exemplified by the changing perception by Maurice Halbwachs - he fundamentally criticized his former teacher - and by Alfred Schütz, whose initially strong emphasis on Bergson retreats already in his first book. ${ }^{9}$ Moreover, the criticism of Bergson's biologist irrationalism

8 On the one hand this is due to the fact that his philosophy of life was adopted, continued or commented on by many thinkers of his time. By Maurice Halbwachs and Alfred Schütz, also two very renowned sociologists count among those intensively adopting him. On the other hand, the Nobel Prize for Literature he was awarded in 1927 made him famous far beyond the sciences.

9 In Halbwachs's case, the more or less explicit distancing from Bergson's doctrines is easier to comprehend than in the case of Schütz. Whereas Halbwachs (1992) eventually only reports on Bergson's point of view as a position which must be refuted by help of Durkheim's terminology - his criticism aims most of all at Bergson's psychologism, which can be traced by his use of the frame concept (see Dimbath 2013) - Schütz refers to Bergson's considerations, which were crucial for his early works (see e. g. Schütz 1982), only as belonging to the preliminaries, to then go on working with Husserl's theory of time and memory. It is remarkable that these two sociologists obviously move away from Bergson's philosophy of life independently of each other. It is improbable that this was sheer coincidence; perhaps it is because in the 1920s, Bergson was still part of the philosophical canon but was considered outmoded. 
appearing soon was so devastating that the whole "system" was no longer perceived helpful. ${ }^{10}$

If Bergson's theory of time and memory, which is rightly criticized, will be taken up again in the following, this is not an attempt at its rehabilitation but for a better understanding of some theoretical aspects - in particular when it comes to the sociology of the 1920 and 1930 s which is relevant for memory research. After presenting some basic motifs, there will be an attempt to identify connection points for a theory of oblivion. On the one hand, this will be done as their well-known motifs of "explaining" oblivion were developed yet ignored afterwards, and on the other hand, because it is perhaps possible to conclude figures of thought which - with all due carefulness - might lead to new interpretations.

One crucial motif of Bergson's philosophy is the concept of continuity, resulting from his criticism of "mathematical time", which is for the first time comprehensively unfolded in Time and Free Will of 1888 (see Bergson, 1950). The starting point for this newly introduced distinction of objective and subjective time is the dualism of life and matter. Life as a force and vital stimulation strives for moving upwards and learns how to make use of downward matter - which is understood to be all that the consciousness perceives as being inactive. Furthermore, the field of life may be distinguished according to two manifestations: plants use vital stimulation by storing it; animals transform it into kinetic energy. This may be continued by differentiating between a human and an animal principle according to which animals, when making use of this kind of energy, follow their instincts, whereas humans tend to use intellect. Moreover, for Bergson, the capability of spatial separation as well as chronological fixation is an achievement of intellect.

Bergson understands space as a quality of matter grasped by consciousness. The consciousness segments reality, which is in a continuous and unstoppable flow, thus creating spatial distances - a helpful illusion that results in theoretical difficulties. On the other hand, time is understood to be an essential feature of life - any life inheres time. However, such a time cannot be "natural" or "mathematical" and outside the consciousness. Thus, this inner time is confronted with "objective" or "mathematical" time, consisting of an accumulation of certain features which are taken out of the "flow". As soon as time

10 See in short: Lorenz Engell (2001). Some early, somewhat sarcastic, criticism came from e. g. Bertrand Russell in 1908, who assessed Bergson's doctrine - in particular his criticism of intellectualism - for being logical and consistent, revealed a number of erroneous conclusions and, as a consequence, denied it almost any epistemic value (see Russell 1946). Only recently, Bergson is read again differently and his approach is considered fruitful (see Ricœur 2004b). 
is understood this way, one will follow the logic of the constitution of space: mental states are lined up or placed next to each other, which develops a well distinguished or distinguishable manifoldness. In other words: this concept of time may just serve as a means for understanding this manifoldness but not for depicting the actual course of time. Among the essence of life, there is also time that is not cut into pieces by way of consciousness processes - particularly the measurement of time. Bergson calls this "true" or "pure" time as continuity (dureé). "Pure duration [...] is the form which our conscious states assume when our ego lets itself live, when it refrains from separating its present state from its former states" (Russell, 1946, p. 796). ${ }^{11}$

Continuity is a format of time in the context of which one attempts to ignore any reflection on the course of time as such. Bergson illustrates this by the example of listening to a melody that is not remembered as a series of notes following one another but as one integrated whole.

Might it not be said that, even if these notes succeed one another, yet we perceive them in one another, and that their totality may be compared to a living being whose parts, although distinct, permeate one another just because they are so closely connected? (Bergson, 1959, p. 10o)

By continuity, no artificial past is construed; rather, it is a merger of past and present within the continuous flow of the I. By pure continuity, the past exists by a present which is constantly reconstituting itself - everything is in progress. That function of the consciousness by way of which past is transformed into present is what Bergson calls memory.

In his 1896 treatise Matter and Memory, Bergson analyses the relation between mind and matter connected to the concept of memory. Firstly, he distinguishes two concepts of memory from each other. The first one consists of motoric processes which have incorporated the past. The other one refers to independent memories of unique, unforgettable events. It is the function of memory to recall past perception contents similar to current perception and thus allow the mind to distinguish before and after (Heinlein \& Dimbath, 2010). There is "no perception which is not full of memories. With the immediate and present data of our senses we mingle a thousand details out of our past experience" (Bergson, 1929, p. 24). The brain is given the task to limit intellectual life to the practically useful:

11 This definition of the concept of continuity according to Bergson is to be found in Bertrand Russell (1946, p. 796). 
The interest of a living being lies in discovering in the present situation that which resembles a former situation, and then in placing alongside of that present situation what preceded and followed the previous one, in order to profit by past experience. Of all the associations which can be imagined, those of resemblance and contiguity are therefore at first the only associations that have a vital utility. (Bergson, 1929, p. 24)

With the help of memory, the consciousness is taken out of the flow of things or necessities. Therefore, it becomes possible to make decisions, as in one single moment, it is possible to intuitively or - in the truest sense of the word "comprehensively" grasp many moments of continuity. ${ }^{12}$

According to Bergson, the subject of perception is impressions or "images" selected by the consciousness. However, also perception as such is already organised by this selection process. In images, the subjective side represented by brain and memory merges with the objective side, i. e., material objects (see Engell, 2001).

By positing my body I have posited a certain image, however at the same time also the totality of all other images, as there is no material object whose qualities, whose definitions, in short: whose existence, is not due to the place it occupies within the totality of the universe. Thus, my perception must be part of these objects themselves; it is rather within them instead of them being within it. (...) Perception would thus mean that my body's possible effect on the objects is detached from the totality of these objects. Then perception is nothing than a selection. It does not create anything; on the contrary, its task is to exclude from the totality of images all those I cannot influence, and then to even exclude from the remaining images all that which is irrelevant for the needs of that image I call my body. (Bergson, 1929, p. 304).

Without elaborating on the cognitive mechanisms with the help of which reasons for such a thesis could be given, Bergson offers an explanation in the sense of more recent schema theories. Furthermore, he combines the selectivity of recognition as performed by memory and a general-universal idea of usefulness. In other words: memory is explicitly not understood to be a place of storing but a time-constituting awareness process by way of which things past are dragged into the present according to "natural" usefulness. The totality of all perceptions and moments exists exclusively in the present; those memories which are not recalled and thus made present at a given moment exist in the same way as all objects of the current environment exist but are not perceived

12 Unlike intellect, which was criticised by Bergson, intuition does not dissect the world into objects - to put it in Russel's (1946) words: it is rather synthetic instead of analytical. 
at this given moment. ${ }^{13}$ According to Bergson, memories are indeed not stored in a specific part of the brain. This insight is found - as stated by Paul Ricoeur in later habitus theories by Erwin Panofsky, Pierre Bourdieu or Norbert Elias (see Ricœur, 2004b, p. 441). The kind of reading represents memories; the body functions as memory.

If memories are no longer considered to be stored "by the brain", also oblivion or other memory disturbances can no longer be ascribed to damaged storage capabilities. Neuropathology knows only the case of aphasia as a disturbance of recognition which must be understood as a motoric disturbance of brain mechanisms - a special kind of oblivion. Although the aphasia disturbance may be connected to injuries of the brain, it is less concerned with a kind of storage. Instead, the brain's capability of establishing a connection between things past and the present is affected. Consequently, memory must be treated as being independent of the brain so that the brain may be understood to be just a communicator between perceptions and movements. In contrast, the memory is corporeal and must thus be attributed to the perception apparatus. In this context, it is remarkable, however, that the brain is said to have an oblivion function:

In this sense, the brain contributes to the recall of the useful recollection, but still more to the provisional banishment of all the others. We cannot see how memory could settle within matter; but we do clearly understand how - according to the profound saying of a contemporary philosopher - materiality begets oblivion. (Bergson, 1929, p. 232) ${ }^{14}$

Memory lives on virtually - if it is not "activated" by the brain, it stays untouched by intentional access and thus unconscious. It is there where Ricoeur identifies a kind of oblivion which does not consist of deleting stored content but is a phenomenon we "may call forgetting in terms of a reserve or a resource". Then oblivion is the covered access to bodily imprinted or rather incorporated memories being out of reach - temporarily or forever - of the consciousness (Ricœur, 2004b, p. 440).

\subsubsection{Internal Time Consciousness}

Edmund Husserl's considerations on the issue of time - like Bergson's - do not deal with ideas of objective time, of a time of nature or of world time. Just as well, he is not interested in the psychological questions of the subjective

13 Paul Ricœur (2004b, p. 412-413) places this insight by Bergson close to Freud's concept of the unconscious.

The final half sentence is a quotation by French philosopher Félix Ravaisson. 
perception of time, such as under which conditions time seems to pass sooner or more slowly or how the subjective perception of time is related to objectively measured time. Instead, his analysis of the concept of time aims at aspects of perception, memory, and expectation, at being time that is no part of the empirical world but results from the immanent time of the course of consciousness.

While connecting to the theory of the origin of time according to Brentano, Husserl illustrates the issue of time as the perception of time with the help of an example: What is astonishing when listening to a melody is the fact that one does not only hear individual notes but that each sound signal still "reverberates" when the next note follows. Without such a "reverberation" of one or even several notes, no "melody" could be experienced.

It is therefore a universal law that a continuous series of representations is fastened by nature to every given representation. Each representation belonging to this series reproduces the content of the one preceding, but in such a way that it always affixes the moment of the past to the new representation. (Husserl, 1991, p. 12)

Both past and future are characterised as modes of time because they change but do not determine. One exception, says Husserl, is the definition of "now". The present can neither be changed nor determined; nothing adds to a perception made in the "now" mode - it is as it is. Referring to Brentano, Husserl states that both past and future are unreal. Only the present is real. In this context, those unreal definitions of time can be in line with just one real one, and the real "now" can become unreal. Remarkably, any real "now" is connected to a necessary before and after. "For it is altogether evident and obvious that everything that is, in consequence of the fact that it is, will have been" (Husserl, 1991, p. 15).

From a phenomenological perspective, Husserl comprehensively criticizes this theory of time, which follows Brentano. However, he says, it is an important starting point. Accordingly, he rejects the idea that a sequence of interrelated events is stored as a sequence by the memory. Rather, he says, the consciousness is just capable of producing comprehensive content at any moment - and just one content. Accordingly, a melody is remembered not as a "chronological series" but as an overall impression, as a snapshot of the complete sequence. For the phenomenological analysis of time, Husserl thus suggests the term "temporal object", which is not only a unit within time but also includes a kind of timeliness. 
That the elapsed part of the melody is something objective for me, I owe - or so one will be inclined to say - to memory; and that I do not presuppose, with the appearance of the currently intended tone, that this is all, I owe to anticipatory expectation. But we cannot be content with this explanation, for everything that we have said carries over to the individual tone. Each tone has a temporal extension itself. When it begins to sound, I hear it as a now; but while it continues to sound it has an ever new now, and the now that immediately precedes it changes into a past. Therefore at any given time I hear only the actually present phase of the tone, and the objectivity of the whole enduring tone is constituted in an actcontinuum that is in part memory, in smallest punctual part perception, and in further part expectation. (Husserl, 1991, p. 25)

This structuring of the audio experience, already announced by the quotation, serves as the foundation for Husserl's theory of inner time consciousness. Each note of a melody - like any other experience of the consciousness "reverberates" within the consciousness for a short time. The reverberation of an original perception is called retention by Husserl. Retention as a form of memory is characterised by soon becoming ever weaker the further away it is from its origin. As long as this phase lasts, we assume that it is still the original sound - thus, the sound, or any other experience of the consciousness, has its own length. "Beforehand' (in the event that was not expected), it is not intended. 'Afterwards', it is 'still' intended 'for some time' in 'retention' as having been; it can be held fast and stand or remain fixed in our regard" (Husserl, 1991, p. 26).

After some time, the retentional consciousness is "empty" again when it comes to this event - a construction that resembles the concept of short-time memory ${ }^{15}$ Retention itself must be understood to be a continuum, in the context of which the consciousness may as well aim at the subsiding retentions and then create retentions of these retentions. Thus, after the original impression, there starts a flow of retentions referring to the original event. At the same time, there is the possibility that second-order retentions develop, for example, when considering the still reverberating note - Husserl calls this process adumbration. Adumbration does not mean that something slowly goes down into the shadows of the past but that an original event, by the process of remembrance, may cast different shadows, each according to the "position" from which light is shed on it via remembrance. Thus, each retention is not just

15 Concerning this idea of inner time consciousness, along the concept of continuity Husserl also develops the concept of the duration of each respective object - thus, he does not agree with the idealistic position according to which all objects are purely spiritual. 
a reverberation of the original impression but may as well become a modification of this original impression.

However, when this first experience is over, the perception experience has not been forgotten. When the note or the melody is over, the awareness of the now-perception comes to an end, and the comet tail of retentions ceases to exist. Now a new phase of remembrance starts. Husserl notes that the memory of a past note is systematically different from retention; in a certain way, it is similar to perception. However, it must be understood as a process of its own that has nothing to do with retentional memory. "Just as I see being-now in perception and enduring being in the extended perception as it becomes constituted, so I see the past in memory, insofar as the memory is primary memory" (Husserl, 1991, p. 36-37). Husserl calls this second kind of memory secondary memory or recollection. Whereas retentional, primary memory necessarily connects to perception events, this is unnecessary in the case of secondary memory.

When we remember a piece of music we have been listening to during a concert, the retention phase is over. However, in our thought, we may once again go through the melody. By recollection, the notes we "hear" in our thoughts, which once again create retentions, melt together with our expectations of those to follow or those still missing. The melody played to our "inner ears" was not "really" perceived; nevertheless, it happened - it was reproduced. Also, in this case, a recollection is required, which covers the whole sequence of notes as a whole. Indeed, then we might play the entire melody in our thoughts by playing the sequence chronologically. However, what is needed for this is an idea or "image" of the entire melody. Recollection appears in different ways: On the one hand, it may simply "appear"; on the other hand, it may be visited purposefully. In this context, the memory may grasp elements of the remembered passage of time or a whole context: we may remember parts of a melody an "earworm" - or remember the entire melody. ${ }^{16}$ As soon as a chronological sequence is recollected en bloc, Husserls speaks of perception. This must be distinguished from perceiving individual notes in the course of listening and the developing retentions. Thus, perception happens both by perceiving the original impression and by reproductive remembrance. For Husserl, primary memory must be distinguished from perception. At the same time, however, each perception creates primary memories again.

Whereas primary memory cannot be influenced, reproducing recollection shows considerable aspects of free decision-making. For example, we may recollect things more or less thoroughly or refrain from further pursuing a memory. Another difference is the fact that primary memory is absolutely safe 
and cannot be distorted. On the other hand, secondary memory reproduces the sequence of events, in the course of which individual elements of the chronological sequence may be emphasized, and others may be neglected or even ignored. However, whereas recollection is still somewhat oriented at the original sequence, a variation that has nothing to do with the primary memory anymore - but may still be imagined - must be called a fantasy.

Another feature of recollection is the fact that it includes expectation intentions that must be met. Husserl calls these expectations protentions. They are empty spaces which, in the course of recollection, are "filled".

Recollection is not expectation, but it does have a horizon directed towards the future, specifically, towards the future of what is recollected; and this horizon is fixed. As the recollective process advances, this horizon is disclosed in ever new ways and becomes richer and more vital. And in this process the horizon is filled with ever new recollected events. Those that formerly had only been indicated in advance are now quasi-present - quasi in the mode of the actualizing present. (Husserl, 1991, p. 55)

Husserl develops a theory of subjective time which is purely based on the individual consciousness. It is characterised by the distinction between sensual perception and inner perception, both of which result in retentions and the possibility to direct contents of recollection in the future even if they are "empty". Against this background, the future is a pre-memory, as only what is recollected can be imagined as an expectation of the future.

From now on, there is no need to pursue Husserl's theory of time further. Like Bergson, he bases the experience of time on a concept of continuity. However, past, present and future are still distinguished as independent realms although the present is reduced to the tiny and fleeting moment of the now. By this theory of time, a theory of memory is formulated en passant - remarkably, Husserl mentions memory only rarely and does so for no theory-systematic purpose. This theory of memory consists of the two components of retention, which is close to experiencing and not to be avoided and reproduction, which only constitutes the sense of time. Part of reproduction is protention, serving as a line of several placeholders which, at the moment of deciding for recollection, are already "budgeted" and are only filled in the course of remembrance. This reproducing recollection is understood to be an independent experience of perception, which again creates primary memories.

For Husserl, oblivion is no relevant subject in the context of his analyses of inner time consciousness. ${ }^{17}$ However, even there, we may find some hints.

17 The concept of the forgetfulness of meaning from Husserl's The Crisis of European Sciences and Transcendental Phenomenology (1970) will be taken up again elsewhere. 
On the one hand, oblivion may happen if there is no reason or drive for recollection. On the other hand, however, recollection includes a certain degree of intentionality or freedom. Oblivion may be calculable by ignoring or leaving out certain aspects of the original experience of the consciousness. However, the reason for reproductions must not be given. This precondition is a paradox, particularly if we make ourselves aware of moments we prefer not to remember exactly. However, protentions are obviously not compulsory and may be filled in quite different ways - with the help of imagination or reduction. As it is a perception experience, possibly purposefully distorted recollection results in new retentions, which again produce foreshadows. Thus, in case of renewed recollection, factually wrong contents are "equally" placed next to factually correct contents. Oblivion is thus a process of the subsequent modification of reality. On the one hand, it may consist of completely refraining from reproduction; on the other hand, it can consist of partial and distorted reproduction. Therefore, the problem of understanding oblivion is not entirely solved, not in the context in which one recognizes something or is told that one has earlier experienced or - in much more detail - known something.

\subsubsection{Oblivion as a Precondition for Remembrance}

In contrast to Bergson and Husserl, Heidegger makes use of a clear concept of oblivion. Following Husserl, or rather independently continuing his thought, he develops a theory of time, which is closely connected to the ontological concept of Dasein. However, his concept is, firstly, considerably different from everything outlined so far and, secondly, it is also quite different from everyday language. How far Heidegger's highly abstract approach may be helpful for a sociological theory of oblivion is the subject of the following considerations. ${ }^{18}$

In his theory of time, Heidegger follows the tradition, from Augustine to Husserl. For example, he discusses his teacher Husserl's distinction between primary and secondary memory and criticizes the idea of linear time this approach is based on. The being of man - Heidegger calls it Dasein - is based on timeliness.

Dasein is characterised by drafting itself or its future. For this purpose, it can only reach back to what it has already experienced and drafted in the past and to what it currently is. In the context of this reference and connecting to

18 The concept of self-forgottenness, which here is presented in somewhat more detail, refers to Heidegger's considerations in Time and Being (1967), published for the first time in 1926. Better known from the debate on French deconstructivism is the concept of the forgetfulness of meaning, based on this debate and developed for the first time by Heidegger in The Anaximander Fragment (1975). 
Husserl's two concepts, Heidegger distinguishes authentic having-been from inauthentic having-been. Authentic having-been is what Dasein is able to retrieve via "repetition".

Inauthentic having-been evades this reflection opportunity of Dasein and, concerning those aspects which cannot be concluded on, is called "selfforgotten". In his analysis of the timeliness and historicity of Dasein, Heidegger wants to discuss the problem of the "deconstruction of a self-forgotten tradition" (Wansing, 2001, p. 256), by positively appropriating tradition first, and then, in the course of reflection, revoking it. Furthermore, Heidegger's philosophy aims at overwriting things past in the course of an effective response. Thus, it is about the project of a deconstructivist foundation of history that liberated from tradition and past and exhausting all liberty possible for men allows for an unprejudiced new beginning. ${ }^{19}$

The distinctive quality of Heidegger's concept of oblivion is that it is not concerned about which topics or contents are forgotten. Rather, oblivion is exclusively referred to as human Dasein. Due to remembrance or, as Heidegger clarifies, re-trieval, we tend to imagine the past based on authentic or inauthentic having-been. However, as images are confused and invented in our minds in the course of remembrance when it comes to things past, we forget what originally happened. Thus seen, any memory is a consequential decision "bought" at the price of far-reaching oblivion.

However, oblivion does not refer to any information but the original, the authentic state of mind. Hence, according to Heidegger, we have no choice but to forget even about ourselves in the course of remembrance. Still, only a fictitious, seemingly gone past develops, which may be more or less accurate or completely wrong. "What makes oblivion haunting is the fact that it allows for imagining something different, for seemingly experiencing a new, inauthentic life" (Motzkin, 1996, p. 182).

Thus, Heidegger puts the well-known figure of oblivion following remembrance upside down and has oblivion precedes any remembrance. The need for remembrance results only from the insights that one forgets and from the search for an own identity, which seems to have been lost in the course of remembrance. Against this background, subject or identity appear only as fiction or as an idea Dasein has of itself. Consequently, it appears as an auxiliary construction by which the creation of the inauthentic in the course of remembrance is supposed to be provided with a positive meaning.

However, for Heidegger, such a way of understanding oblivion leads to his own, oblivion-related theory of time. It is possible to establish a relation to

19 So far, compare this to the overview by Rudolf Wansing (2001). 
the past in the sense of "authentic" if it is both repeatable and never gone. As soon as the past is understood to be gone and no longer possible, it is indeed no longer relevant. Then, one no longer focuses on oneself and the authentic past but the nearest things in the world. For this, Gabriel Motzkin finds the following interpretation:

We consider the past void because we consider the present something we might lose, thus a lost present. By forgetting the past we have also already forgotten the present, and we have never remembered the future. This indifference towards of the present makes time crack, and instead we consider the world the nearest instead of ourselves. (Motzkin, 1996, p. 188).

However, Heidegger's philosophical concept of oblivion, which refers to human Dasein, appears to be sociologically irrelevant at first sight, for Heidegger himself states that men's dealing with reflecting on Dasein seems to change through the ages and cultures. In modernity, thinking emphasises the present, which is the same as increasing oblivion. When it comes to time and history, Heidegger concludes from this the demand for deconstruction, to control the inauthentic and to keep all possibilities open for the future. The oblivion problem formulated by Heidegger can be "sociologised" against the background of the thesis of a change of mind or how humans think. Sociologically relevant is also the fact that he understands the process of oblivion as an intentional activity, as action. Thus, oblivion does not appear as an unintended side effect of remembrance but is the responsibility of the individual that approves forgetting the authentic, quite purposefully by remembering in a specific way.

\subsubsection{Traces}

Referring to Heidegger's philosophy, Derrida demands to enlighten everything metaphysical through critique and critique of existing critique. In this context, the method of deconstruction he developed may be considered a process of recalling. What is supposed to be remembered is the historical development of texts which are all too quickly objectified. ${ }^{20}$ Deconstructivism uncovers formal logical contradictions by considering and judging a subject, taking different perspectives or a thorough analysis of the context (Weinberg, 2001). ${ }^{21}$

20 By text Jacques Derrida does not only mean anything written but any kind of linguistic statement or statements changed into language. A further analysis of the of the trace is presented by Jean Greisch (2004), among others.

21 This is not, like in Heidegger, about destruction - Ze'ev Levy (2007) points out to the syllable "con" providing a hint at decomposition and dissolution, from which a trace becomes visible. It is not the task of the trace to reproduce that what the trace has left behind. "The same holds for science: a fossil is a trace, but who has left it has completely disappeared" (Levy, 2007, p. 150). 
The method of deconstructivism accepts no presupposition concerning any problem - usually a philosophical one. Even the presupposition is nothing other than a trace indicating the subject under consideration. Any subject results from the difference it creates regarding everything to which it might be related. However, this difference is not only produced as a distinction from spatial or factual perspectives but also as a reference to the timeliness of a subject. Thus, even earlier states are nothing other than traces indicating a current state.

For the deconstructivist, any assumption of equality is suspicious. Most of all, when it comes to theories of timeliness - in particular the phenomenological ones -, deconstructivism points to the problem that a subject's present cannot result from the retentions and protentions concerning it. In the case of a synthesis of retentional and protentional traces, part of the being under consideration is necessarily overlooked, which is demonstrated by Derrida by referring to the concept of the unconscious in Freud. The unconscious, he says, is no horizon of changed presents but a "past" that has never been present and will never be present (see Derrida, 1982). It differs from the present by postponing itself, overcoming the alternatives of presence and absence while at the same time, however, sending out representatives to attract attention.

Deconstruction is a method for "working on" the problem of the oblivion of being as formulated by Heidegger. However, the oblivion of being, that is, forgetting the difference between being (Sein) and essent (Seiendem), between being present and that what is present, can only be experienced as having been forgotten if it reveals itself in a trace. Otherwise, the essent stays hidden within being - which is, after all, a problem of reification or inauthenticity.

There is no need to pursue Derrida's thought further, especially as the deconstructivist analysis refers first of all to the philosopher's work. However, in his work, we find another development of Heidegger's considerations that may be useful for a sociological analysis of oblivion. At the heart of his theoretical work, there is the concept of trace, which is commonly considered a reference to a subject belonging to its context but comes with the problem that expiring is part of its structure. The trace is ephemeral, yet, of crucial significance: The present "is a trace, and the trace of the erasure of the trace" (Derrida, 1982, p. 24). In other words: it is possible to conclude on a present or current subject only if it is considered by its difference to its context. Each of the possible differences is a trace that falls into oblivion again as soon as having been grasped. In this context, the remembrance work of deconstructivism happens beyond the sheer meaning of the term, as all that might be "reconstructed" this way is being but not the authentic essent which is "real" in the current context.

Before the following genuinely sociological theories of time will be discussed to identify starting points for an analysis of oblivion and clarify the concept of 
social memory, the thus far collected philosophical hints are briefly assessed. The first philosophy of time discussed in more detail is Bergson's philosophy of life. Bergson is the most prominent representative of more recent theories of subjective time. It was most of all he who introduced the concept of inner continuity, which was so influential for subsequent sociological works. The then presented approaches belong to one developmental context, as they all come from Husserl's phenomenological school. Husserl's theory of inner time consciousness - time is grasped as a transcendental phenomenon resulting from remembrance processes - is their starting point. For the philosophy of Heidegger, who was Husserl's disciple, time is a crucial topic of his philosophical work, which, however, can only be cursorily touched here. Nevertheless, in Heidegger's work, we find an oblivion theory of its own kind, which can also be sociologically interpreted as the issue of the oblivion of being. This is where Derrida takes up Heidegger's thought and attempts to explicate the latter's deconstructivist way of proceeding. Also, the only constituted school of deconstructivism may be understood in the context of memory-theoretical signs. However, for an analysis of oblivion, it is most significant when it comes to the concept of trace - a concept we are going to come back to elsewhere.

For a start, our look at philosophical theories of time has provided some insights into the close connection between time and memory or remembrance. The time of consciousness develops from the capability of remembering something. In this context, time appears as a theoretical aid for transcending thought, hence, becoming capable of making drafts and being capable of action. However, the reason for developing a time consciousness is - at least according to the current state of the unfolded positions - maintaining and organising experiences as a general experience that is always immediately over. On the other hand, oblivion appears as a vast "shadow" absorbing any kind of experience that cannot be temporalized as an experience. Thus, we would have to say that not only remembrance but most of all temporalisation constitutes the reflecting of the consciousness on the past. Only this way it is possible to prevent some knowledge from falling into oblivion entirely.

\subsubsection{Time in Sociology}

There are only a few approaches to a sociology of timeliness, both in the theoretical arsenal of general sociology and in the vast field of special sociologies. Given the ontological "status" of the concept of time, this does not come as a surprise. Whereas for sociologists, action theories are as essential as structural theories, theories of time are marginal when it comes to the fundamental orders of the discipline's concepts and theories. At a closer look, however, one must admit that almost any sociological theory deals with the issue of 
timeliness. Most of them more or less consider time to be given, and often the representatives of theories or those applying them are not able to elaborate on time. $^{22}$

At first sight - and in line with approaches to the philosophy of time of the humanities - one agrees that some mathematical, natural-scientific or objectivist concepts of time are mostly unsuitable for sociological purposes, ${ }^{23}$ as each of the different phenomena of the social unfolds its structure of time.

However, the impression that sociology is not very sensitive to dealing with time theoretically can only be confirmed for certain fields of sociology. Both among the "classics" of the discipline, general sociology and sociological theory, we frequently identify relevant considerations. For Durkheim, who refers to Aristotle, time is one of the fundamental categories of thought. Accordingly, he says that it is impossible to grasp any subject theoretically without fixed regulations of time. ${ }^{24}$ Sometimes even sociological theories in the context of which no independent concept of time is constructed include at least hints at "classical" theoretical references providing approaches to a clarification. We seldom find references to the issue of time in studies on social change or in the context of the diagnostics of the time. ${ }^{25}$ Precisely in this area in which sociology must be crucially based on timeliness, there seems to be a reflection deficit; perhaps it has simply been forgotten that also the chronological frameworks of their diagnoses must be based on reasons.

If there is a stock-taking of sociological theories of time in the following of both those theories explicitly formulated and those implicitly assumed -, the focus needs to be on the function of a concept of time for the theory of social memory remembrance and oblivion. For as soon as one speaks of stability or dynamics, of structural maintenance or structural change - no matter in which ways - this includes questions of social memory, remembrance as well as social oblivion.

22 It is this way in which Armin Nassehi (2008, p. 35-36) starts his analysis of the issue of time in sociology, by pointing out that sociologists readily agree with the statement "All time is social time". During conversations, however, he says, one frequently encounters helplessness when addressing the issue of time.

23 This insight was formulated already by Pitirim A. Sorokin and Robert K. Merton in 1937.

24 Exemplarily, Émile Durkheim (2008, p. 11-12) proves time to be a social issue through and through, which helps thinking with regimenting individual memories into a collectively binding scheme or image of continuity. The structuring of time by a civilisation - such as the calendar - constitutes from the rhythms of collective life.

25 For example, Stefan Böschen and Kurt Weis (2007, p. 23) point out to the fact that such an "inspiration" is obviously not the "rule" although, due to the temporariness of conflicts between institutional fields or fields of action, it is imperative. 
Through the previously sketched philosophical theories of time, it has already become evident that there is a close connection between time and memory or remembrance. Also, those pioneers and founders of sociology, which are considered classics, were inspired by these models. However, there is a particular difficulty in transferring concepts of time that were developed in the context of the individual consciousness and its cognitive faculty to the realm of sociological empiricism. Perhaps the necessary reorientation of the issue of time at sociological questions marks the beginning of the "separation" of the issue of memory.

Flipping through sociological works for their specific ways of dealing with the category of time brings us to the early works of US American Pragmatism and sociological systems theory (1). Peripheral areas of sociological thought, mainly circling the constitution of the present, are the special sociologies of historical sociology and the sociological research of the future, which here can be discussed only marginally (2). More visible is the sociological interest in measuring time and grasping time and the resulting consequences for social relations (3). Then one last point is dedicated to time in the context of theories of social change (4). Each of these approaches provides hints at the issue of oblivion. Thus, in the following, it will not be about general considerations on a sociology of time. ${ }^{26}$ Instead, the focus will be on a reconstruction of the issue of time, based on the theoretical canon of sociology, in the sense of an intermediate stage on the way towards a general stocktaking of possible perspectives of social oblivion.

The first one of the unfolded theoretical sociological perspectives on the issue of time is more or less immediately inspired by the philosophical insights on inner continuity or on inner time consciousness and comparable analyses from the context of US American Pragmatism. The starting point is the question about the mutual conditionality of past, present, and future from the individual's point of view. The genuinely sociological contribution refers to how these insights have a social effect and may constitute intersubjectivity and sociality. Still, the analysis will be based on a diachronic, process-oriented perspective or understanding based on continuity. The social philosopher George Herbert Mead bases his considerations about the nature of the past on the theory of time developed by philosopher Alfred North Whitehead and Bergson's theory of memory. For him, the past is represented by memory images contributing to constituting the present. Thus, the place of the past is

26 Sociological considerations on the topic of time and time-sociological transfers onto research fields of sociology are to be found in the works by Werner Bergmann (1983), Günter Dux (1992) and Helga Nowotny (1992). 
not in the past; it is - by making oneself aware of it - an element of the present. The experience of time results from the continuous flow of experiences, in the context of which the consciousness faces the difficulty that this continuity must at first be produced. Thus, it "questions" each new impression for its identity or its difference from existing memory images. "That which is novel can emerge, but conditions of the emergence are there" (Mead, 1964, p. 236). That imaginations - of things past - may be chronologically organised cannot be explained by the laws of natural-scientific space-time. It is a sheer achievement by the consciousness.

For Mead, ideas are imaginations that can be organised continuously. Everything else he calls a fantasy. To perform the constant comparison of what is remembered and new impressions, Mead extends the phase of the present as far as to a constant overlapping of the current and the past. However, the assessment of continuity always identifies discontinuities. Then the consciousness starts with constructively reconstructing continuity. In this context, the past is usually adjusted to the present. "The past is what must have been before it is present in experience as a past" (Mead, 1964, p. 238). By this insight, Mead criticizes Bergson's theory, according to which the administering of the constantly rising flood of stored memory images belongs to memory functions. Rather, Mead says, continuity is produced as an achievement by the consciousness. This way, he connects to Kant's considerations, according to which an unorganised sensual content becomes experience as soon as it has been transformed into categories of reason. Accordingly, the transition from present to present - in the form of difference - may always result in something new. The inclusion of the past does never produce anything new. On the other hand, the past connects the subsequent presents by making one present dissolve in the subsequent one.

Future develops when the consciousness tries to prolong, as fantasies, the continuities it believes to identify in the present - however, thereby, it stays hypothetical and can at best place the new of each respective present in a row it has already "anticipated" in such a way as to cause the impression of causality. As a consequence, there results that even scientific insight is merely a construct. For example, the historical sciences construe continuities based on historical sources. Then these sequences of events are ascribed to certain people living at certain ages. However, they have not necessarily got anything to do with how the historical actors perceived the sequence of events. This construct assumption also holds for natural or natural-scientific time. Mead demonstrates this by an example: just a minor change of the spectroscope may add to or take away some millions of years from the lifetime of the stars (see Mead, 1964, p. 240). This makes him state that the validity of a seemingly 
objective past is based on the continuity of its structures. In other words: there is no history of presents. Always the past develops - artificially - under the impression of a new problem. It is essential that the continuities this is based on are discovered by what has recently developed. However, they will be valid only as long as some novelty demands another history. "The past thus belongs to a generalized form of experience. It is the arising of relations between an emergent and a conditioning world" (Mead, 1964, p. 242).

Mead's time-theoretical position is in stark contrast to the essentialist idea of an objective or natural time. It is wholly tied to structures of the consciousness and emphasizes the constructed nature of memories. This insight, which is at first an egologic-psychology-of-consciousness one, may well be transferred to the realm of the social. For example, the same principle of continuity as a construct may also be assumed for supra-individual remembrance - a both relativistic and at the same time presentist position which abstains from any way of fixing the past in the past as a basis of both the subjective and the social genesis of knowledge. While doing so, Mead does not rule out that there might be experiences that are stored by the consciousness. However, only by being updated, they become an interpretation of the present, and they are activated only to create continuity. Moreover, it seems to be evident that the already proven patterns are used for this continuity production.

If memory is just a construction when it comes to continuity - and thus connectivity - in the sequence of presents, the issue of oblivion seems to stay in the dark. However, from the presented arguments, we may conclude that oblivion happens either where it is impossible to construct continuity and where existing experiences prove to be no longer adequate. Alternatively, it happens where the experience of the new includes more than necessary for the construction of continuity. Thus, oblivion either happens if the past is no longer sufficient or if the stock of things past is satisfied all too soon.

Even if certain cycles of time exist both outside the psyche and outside the social, humans create their own time, which usually corresponds with the aspects of "natural" time. Social time, however, only develops by the institutionalisation of certain regularities as binding patterns of orientation. The individual may indeed live entirely according to his/her ideas of time; still, he or she must expect to disappoint the expectations of others and perhaps be sanctioned. This social time diktat is systematised and increased by the measurement of time. Thus, what is sociologically relevant - and there Halbwachs stays obliged to Durkheim's thought - is only the organisation of time as developed from social evolution. By this concept, Halbwachs at first distances himself from Bergson's understanding of inner continuity, which is of a subjectivist nature in his opinion. Only with the help of time conventions, 
it becomes possible for different individuals of different ages to reference their actions to a time - otherwise, a baby and an old man would refer to completely different subjective perceptions of time, and no adjustment would be possible. However, this step towards institutionalised time results in further consequences. For example, also different social groups reach back to different concepts of time. The time of each group results from its stock of artefacts its memory is oriented at, thus creating time. Groups or cultures with only a few objects to be attributed to their past are thus provided with only a smaller window of time. Halbwachs states that social time as historical time can only grow from memory. A time which cannot be "hinged" to memories is - and here he is in line with Mead's concept of time - either non-existent or irrelevant. Also, historicising reflection happens according to this mechanism. However, explicitly dealing with traces of the past construes a time of its own which has no longer much to do with the social time of its culture. Vice versa, time becomes itself a memory aid by serving for visiting specific memories as soon as it has been intersubjectively fixed: we may make the time of a group our own time by e. g. remembering a conversation with classmates - in our minds, we are back at school again.

Time is real only insofar as it has content - that is, insofar as it offers events as material for thought. It is limited and relative, but it is plainly real. Moreover, it is large and substantial enough to offer the individual consciousness a framework within which to arrange and retrieve its remembrances. (Halbwachs, 1980, p. 127)

According to Talcott Parsons, the structuralist-functionalist theory is about reconstructing universal structural aspects that are thus valid for any society or necessary functions. This theory does not aim at any clear concept of time; however, it requires explaining how social structures succeed with surviving over time. For this, Parson's systems theory provides the field of culture or latent pattern maintenance. ${ }^{27}$ If, however, a sub-system of its own is needed, which guarantees or legitimates the continuous existence of a structure, this system must be constantly irritated, which does not suggest stability but rather change. In other words: the function of pattern maintenance implies an understanding of the process or assumption of change which cannot do without an idea of time.

Social systems are subject to a continuous adjustment process. Parsons describes this process as evolutionary change happening most of all by functional differentiation. The aspect of time characterises a process in which a

27 In the context of the concept of social memory see Marco Schmitt (2009), among others. 
simple state of the system results, due to change, in a more differentiated state of the system. The present annuls the past, the new proving to be differentiated and now well distinguishable functionality.

Regarding social progress, Parsons explains this with the help of three evolutionary steps (Parsons, 1966). The transition from a primitive to an intermediary level first consists of the development of language. However, only as a result of introducing written language, the social system can be distinguished from the cultural system. "Only literate cultures can have a history in the sense of an awareness, based on documentary evidence, of past events which are beyond the memories of living persons and the vague hearsay of oral traditions" (Parsons, 1999, p. 38).

The step from the intermediary to the modern level happens in the course of developing institutionalised codes within a legal system of normative order. The particular step to modernisation consists of the law becoming independent of the normative components, such as political or economic interests.

In contrast to theories of a specific historical development, Parsons is interested in developing a general theory of social evolution. Accordingly, he states that "historicism has characteristically denied the possibility or relevance of generalized analytical theory (which systematically treats the interdependence of independently variable factors) in explaining temporally sequential socio-cultural phenomena" (Parsons, 1966, p. 127) at the end of his comparative study on "societies".

Time emerges as a variable of general evolutionary differentiation processes abstractly formulated and stripped of the concrete aspects of past events. These processes do not even stop at the cultural system, and pattern maintenance should not be understood as a solidification of structures, which is a matter of course against this background. In his theory of patterning, Anthony Giddens combines the action level and the structural level. According to his accusation addressing functionalism and structuralism, this theory paints a much too static picture of society without sufficiently reflecting on the problem of time and timeliness. He states that through synchronous structural analysis equating time with change, it is impossible to reflect on the aspect of time, which is constitutively included in any action and any social system. Whereas Giddens's temporalisation of structure is criticised as an attempt to fix an outer frame, the evolution-inspired revision of structural functionalism, which Giddens perhaps ignored, is said to allow for a higher level of reflecting on social time and timeliness. ${ }^{28}$

28 These ideas are also to be found in Werner Bergmann (1983) who refers to Heiminio Martins in this respect. 
An interpretation of the issue of social time, which is completely different, perhaps not concerning its theoretical points of reference but in its theoretical architecture, is offered by Niklas Luhmann when it comes to the development of a systems-theoretical concept of time. At first, he states, as a feature of the social construction of time, that on the one hand, time must not be confused with the computation or measurement of time. On the other hand - and this is a diagnosis-of-time view -, social time is characterised by, the present shrinking to being just one point or being the difference between past and future in modern society. In contrast to Aristotle's concept of time, Luhmann states that this development is due to the discovery that there cannot be any origin of the past and, by consequently transferring this insight to the future, also no "end of time". In other words: past and future are extended into infinity, and at the same time, the present is reduced to marking the turning point between the two. Accordingly, topicality must be imagined as immediate change, and time itself as an "interpretation of reality with regard to the difference between past and future" (Luhmann, 1976, p. 135).

This originates from the idea that any system always only operates in the present by being different from its environment. However, if time develops from the difference between past and future, the system observes its reality with the help of this distinction. It is about the difference of the system's current state from possible alternative states of the system. The change from one state of the system to another, which happens in the course of observations, takes time. At the current moment, the system reflects on past and future, which appear as two horizons demanding selection - or a decision. When construing past in its present, from various possibilities of what has been, it selects those possibilities as relevant for the system and construes its own history. Moreover, the projection to the future happens according to this pattern: only what is currently relevant for the system is construed as the future. Thus, time is just imaginary for the system and has nothing to do with any material environment or objective time. "The passing present allows for the reversibility of the selections and most of all for the contrast to the punctual present which allows for experiencing continuity and a feeling of time passing by" (Baraldi et al., 1991, p. 216). ${ }^{29}$ In this sense, time consciousness develops as an answer to the necessity of imagining consistency and change simultaneously, as the precondition for selectivity in the relation of system and environment (Luhmann, 2009, p. 136).

29 See the glossary on Niklas Luhmann's theory of social systems, keyword time (Baraldi et al., 1991, p. 216). 
Elsewhere Luhmann points out the close connection between time being construed by the system and the system's memory. In this context, memory is defined as a reflection authority that is free to make disappear what is happening or to connect it to schemes in the mode of observation. However, also the event nature of time itself must be understood as a scheme in this context (Luhmann, 1996).

If Luhmann's concept of time is stripped of the motifs he believes to be necessary for the development of the theory of the social system - such as the problem of the permanent development of differences or the co-presence of simultaneity and non-simultaneity - it becomes evident that his concept of time is conventional beyond the genuinely systems-theoretical problem. Like Schütz, he deals with creating continuity from a (systems-)egological point of view. As his theoretical perspective excludes the category of the subject, he can circumvent the problem of intersubjectivity. If time is always constituted from the perspective of a closed and self-referential system, no chronological horizon of a system must be analysed by referring to other systems. The social system creates its own time, just like the scientific system, the organisational system or the psychological system. Accordingly, the problem of synchronicity is shifted into the system - such as into figures of co-presence. A "true" parallel to the question of intersubjectivity develops only if two social systems, which are open towards the world yet operatively closed, start interacting. First, Luhmann discusses this as interpenetration; however, he replaces this term with structural coupling later. Now structural coupling - e. g. as the interaction between a consciousness system (consciousness) and a social system (communication) - is not understood as a causal relation of mutual determination but in the mode of simultaneity. However, the simultaneously produced results are processed by the involved systems according to the appropriate systemimmanent logic so that no "material" intervention of one system in the other happens. ${ }^{30}$

30 It is remarkable that supporters of the theory of social systems in Husserl and most of all in Schütz make the lack of a theoretically-systematically satisfactorily solution for the intersubjectivity problem one of the main arguments of their critique, to then, by making use of a number of abstractions and reformulations of concepts, introduce the concept of structural linking. Such a concept can then be discussed by help of the same arguments as the positings and general theses of the phenomenologists. "If communication and consciousness are each imagined as autopoietic systems, both the results gained from Husserl's and Schütze's insights on the structure of inner continuity can be adopted and the necessity, explicated by Mead, of imagining an operative unity for the description of social time whose elements are social entities and not elements of the consciousness can be fulfilled" (Nassehi, 2008, pp. 178-179). Quite obviously, what is presented here is not an independent answer but just a reformulation. 
In particular, in the context of French sociology - again, while connecting to Durkheim's attempts to establish sociology as an independent scientific discipline further - there were attempts at delimiting from the historical sciences. For example, the debate between Halbwachs and historian Marc Bloch on the difference between social memory and history as a crucial inspiration for the sociological reflection on time may be considered in dealing with the past. ${ }^{31}$

Already here, it becomes apparent what Luhmann is going to state at a later time: in contrast to historians, sociologists are not interested in the contents of the past but in the selection or structuring performances connected to the past. In this sense, Rainer Schützeichel states in his characterisation of the tasks of historical sociology: "Sociological information about social situations by way of reconstructing their historical genesis" (Schützeichel, 2004, p. 9). ${ }^{32}$

One binding motif of sociological theories of time is the analysis of how individuals and society are related to the future. On this, Werner Bergmann states that sociology has increasingly been discussing the problem of orientation in the future, most of all against the background of its significance for social behaviour and decision-making. For a "classical" motif in the context of social stratification, he points to the research of the group-specific readiness for deferred gratification (deferred gratification pattern). Particularly in social planning, sociologists have been dealing with time orientation and timeliness (Bergmann, 1983).

Luhmann, who understands future as a horizon of the present, concludes that future cannot start, referring to Husserl's phenomenological concept of time. Just like we will never arrive at the horizon, the future will always move away from the observer and has only an orientation function, after all. In contrast to the philosophy of time in antiquity, Luhmann states that the possibilities of the future, which is now basically open, must be reduced by moving towards it. ${ }^{33}$ Accordingly, the evolution of time-consciousness must first be understood, by futurization, as an extension of the future towards a horizon of open possibilities. The alternative - that is, the resulting necessity to reduce open possibilities - is called de-futurization by Luhmann. Under conditions of growing complexity, there is a growing need for temporal integration. This is where Luhmann introduces a modulation of the concept of future: the current future and the future present. The current future is a projection surface

31 Halbwachs (1980, p. 57) comes to this conclusion: "Our memory truly rests not on learned history but on lived history."

32 Now, starting out from this definition, Rainer Schützeichel (2004, pp. 9-13) expands the task field of a historical sociology onto all fields of sociological research which are in any way associated with the chronological categories of past and present.

On this as well as on the following see Niklas Luhmann (1976). 
for utopian and dystopic thought, whereas the future present is determined by technology and planning. Utopias are characterised by "pushing" the present future ahead of itself; the future cannot begin because it is always imagined from the point of view of existing structures. These structures must be changed before utopia may become a reality. Planning and technology de-futurize the future horizon; this happens by interpreting the present as the past of a future present through statistical calculations and predictions. Defuturization bereaves the future of its openness and uncertainty (Bergmann, 1983, p. 469).

Defuturization results in forgetting about possibilities, as most basically plannable scopes for design are ignored and no longer recognized. Given the contingency concept, which includes all possibilities of future actions in the form of the lifeworld horizon, the problem of oblivion covers only those possibilities which have been taken into consideration as drafts but have never been realised.

A sociological theory of time which understands itself to be a sociology of knowledge was developed by Norbert Elias (1992). For Elias, not the question of how consciousness constitutes time is at the fore. Instead, he is interested in the ways in which societies practice time over the changes of history by consequently delimiting from the concept of time of the natural sciences. In Elias, time as an orientation category is connected to power, and power - which we learn from his analysis of the process of civilisation - must be considered a historical process (Elias, 1981). He pursues the goal of a historical analysis of social ways of dealing with time due to the assumption that developed or civilised societies tend to forget about their time being a construction, of orienting at natural-scientific ideas of time and of even hypothesising the latter as a universal idea. Reification happens due to the individuals orienting at standardised time continuums such as time on the clock - at first by external compulsion, then as self-constraint: in progressed societies, the capability of being socialised as a self-constrained is an element of adult life. Elias, who understands time as a symbolic order based on power, after all, is interested in comprehending the development of social knowledge. In this context, time has "only" the status of a concise case example in the context of which no man is the subject of knowledge but the flow of generations in the course of the development process, i. e. humankind as a whole. ${ }^{34}$

At the heart of Elias's analyses, there is no explanation of time as producing a continuum and not explaining simultaneity. Rather, his starting point is a synchronicity to demonstrate the integration of a socially construed and, over

34 We may thus - and this was probably inspired by his teacher Karl Mannheim - speak of a a synchronicity problem in the sense of a simultaneity of the un-simultaneous. 
the centuries, incorporated symbolic order. Issues of memory, remembrance or oblivion are hardly touched - except for the question of what is forgotten if social life is exclusively oriented at standardised continuums of time. The time symbolism of measured time, however, is imagined as a power-induced principle of social selection which, on the one hand, guarantees integration by way of producing simultaneity and liberating interaction from the immediacy constraint - for example, one can make appointments. On the other hand, standardisation makes us forget that some social subjects cannot be organised according to standardised time.

As a result of establishing the practice of time measurement, individuals and collectives are confronted with new problems when it comes to reflecting on things. Units of time and time schedules indicate experience gaps, in particular in retrospect. The experience of having lived through rhythmed time without being able to remember several individual moments indicates the fact that obviously, one has forgotten about certain experiences. A time consciousness that is exclusively constituted via experiences and does not have to relate to "objective" methods of time measurement is less confronted with this problem. It may be that the day planner is not just connected to de-futurisation but that it is also an invitation to reflect on - perhaps socially relevant - empirical knowledge in the context of appointments in the past.

It has already been pointed to time being the precondition for any consideration on social change; however, it is also the precondition that theories of social change rarely refer to time and timeliness theoretically. Max Heirich carried out an early analysis of the straightforward yet frequently implicit ways of understanding time as found in models of social change. Heirich identifies four ways of reading time, the first of which is understanding time as a social factor or bearer of social significance; the second one is understanding time as a link to other variables in the context of social sequences; the third one is understanding time as a measure in the context of the measurement of changes; finally, the fourth one is understanding time as a qualitative indicator for the change of social structures. ${ }^{35}$

One prominent representative of action theories, who presents an implicit concept of time, is Max Weber. As it is well known, Weber's meaningadequate sociology looks for answers to how social phenomena have developed historically. Accordingly, Weber derives his thesis of the connection between the Protestant ethic and modern capitalism from historical analyses. Understanding comes from comprehending possible developments resulting

35 On this see Werner Bergmann (1983), and also Max Heirich's (1964) essay on time and social change. 
from certain social phenomena of the past. Such a historically reconstructing method at first suggests - in particular by its condensing perception - a reduction of the process-oriented argument to two points in time: a past, in which everything was different, and a today.

In Weber's works, the construction of the reasons for action delineates an implicit developmental logic. For example, instrumentally rational action as a feature of the modern world may be confronted with the value-rational and traditional behaviour of a past world. In the latter case, the wording alone - indeed Weber does not speak of routine-guided action but purposefully emphasizes tradition - indicates the time-diagnostic intention of the typology. Also, the ideal types of authority relation may be interpreted similarly. The rational, legal, bureaucratic rule results from a rationalisation process starting from older ways of rule. For his pessimistic predictions concerning the consequences of modernisation and rationalisation processes, Weber uses catchy formulations, such as the "shell of bondage" or the dystopic characters of the "specialist without spirit" and the "sensualist without heart". When it comes to dealing with time, he applies a practice that, as far as so-called diagnoses of time or the present and adequately formulated predictions, does mostly without any definition of a concept of time. The fact that sociological predictions may be successful even without such clarification does not justify giving up on it, as one or the other statement would certainly become more precise with the help of an elaborated concept of time.

Concerning time-based ideal types, precisely in view of the modernising shift of emphasis, e. g. from value-rational to instrumentally rational behaviour, an oblivion process may be diagnosed, which first of all covers the subjective meaning of action but not practical action as such. The this-worldly asceticism of the Protestants, which may at first be based on value-rationality and may indeed be interpreted according to instrumental rationality, is forgotten in the course of secularisation processes. The only remaining legitimate reason is that of instrumental rationality - in this case, the action practice survives the motivation for action. Finally, this results in a situation of forgetting about the meaning, which is not necessarily perceived in practice and is first of all of interest for the conceptual historian. In this section, after having presented an overview of philosophical theories of time, some always related concepts of time coming from sociological theories have been analysed. For an analysis of oblivion with sociological concepts of memory and remembrance, the concept of time is fundamental, in so far as it provides a frame for the possibility that a stock of knowledge can be taken out of the consciousness or simply disappear. In particular, a look at the continuity problem of social timeliness makes it obvious that in each case, time is a current tool for orientation 
or aid for combining the currently new with what has been and for creating identity. In this context, oblivion is always mentioned if the interpretation of the present does without certain schemes representing the past. The insight that something has been forgotten can only come from being confronted with an incomplete representation of the past. And obviously, this happens only in a situation of communication or interaction, that is, when participating entities interpret the present simultaneously and refer their interpretations to each other - we may say: individuals. Also, concerning the ways of understanding timeliness implied by sociologists, we may state that the use of time as an orientation scheme frequently refers to the problem of remembrance or the selectivity problem coming along with oblivion. In how far, after all, oblivion just refers to the waste products of selections or must be understood to be an active element of constituting the present in this context will have to be clarified in the further course of the here unfolded considerations.

When comparing the philosophical and sociological concepts of time to theories of memory, it is striking that both are much dependent on each other. Without memory, time can neither be experienced nor construed and vice versa. It would be pointless to imagine memory if we had no time consciousness which at least marks the difference between a before and an afterwards. The concept of time is as essential for memory and for the problem of remembrance, as little as it is connected to oblivion. Even figures of speech such as that of the "ravages of time" rather address memory, to which a currently perceived decay is contrasted. However, as demonstrated by the considerations on the connection between time and oblivion so far, eventually, time is constitutive also for oblivion - in how far this works vice versa cannot be discussed here.

\subsection{How Does Oblivion "Work"?}

In the brief considerations on the connection between knowledge and oblivion presented at the beginning of this book as well as in the detailed excursus on the issue of time, the concept of oblivion has already been comprehensively applied. However, what is still lacking is the assessment of the concept - fundamental for any knowledge-sociological approach - along with the range of its everyday language and scientific meaning. The second step of approaching the concept of oblivion will be a meaning analysis in different contexts. What do we mean when speaking about oblivion?

Forgetting an appointment, the keys, your PE kit, your homework, losing your train of thought - all of these too familiar situations, which we are sometimes reproachfully reminded of, are deeply rooted in our everyday lives. 
Sometimes, however, we are also told to "forget about something". This is either meant to say that the concerned subject is unimportant or unachievable for the time being. These two everyday ways of making oblivion a topic of discussion both offer an unexpected insight into non-availability. It is about something we believe to need now; however, it has either been lost in the past or is prohibited by its future use.

Having forgotten a name, relatives, the way home, to drink, to dress - against the background of improved medical supply and thus growing life expectancy, the fight against the pathological loss of the knowledge of vital performances and social relations appears as a new challenge to modern society. The growing significance of dementia makes the problem of oblivion socially relevant. At the heart of these phenomena, there is the unacceptable loss of knowledge. Oblivion is understood as a deficit; what has been forgotten is considered something that may be expected under normal conditions.

Having forgotten the culture, the tradition, the origins, and the purpose also motifs of cultural criticism are connected to lost knowledge. Usually, processes of modernisation, rationalisation or differentiation are said to be responsible. Sometimes, however, oblivion is longed for: if criticism does not refer to dangerous knowledge but traditional knowledge or to the fact that sometimes we must even be able to forget something: Forget it!

Already a brief look at different situations of making oblivion a topic of discussion makes evident that the loss of knowledge is mainly perceived as a deficit - this applies even to the lonely moment at the door at night. Given the many problems oblivion seems to cause, we tend to forget that oblivion has important functions both in the neurophysiological, psychological and social context. It helps to ignore what is unimportant and thus stay capable to act.

Thus, what is the meaning of oblivion? Must oblivion be fought? What would be the consequences of a victory over obliviousness? Or should we rather try to understand the phenomenon of oblivion better, to be able to estimate the advantages and disadvantages of the loss of knowledge or of giving up on it? Hence, it is nearby to provide information about the contexts within which oblivion is discussed first. This will be done in the next section, starting with an assessment of hints at oblivion as they are formulated in encyclopaedias and found in everyday language. Then, a more detailed assessment of spotlights on the cultural-historical way of dealing with oblivion will add to this passage. Finally, a classification of the ways and functions of oblivion will be presented.

\subsubsection{Oblivion in Everyday Language}

In a german dictionary, under "vergessen" we find the phrase "losing something from our (intellectual) property" (Duden, 2007, p. 1805). The formulations offered by the related terms dictionary by the same publisher on the 
one hand refer to the concept of memory: "losing all memory of something". On the other hand, we find the formulation: "Not (no longer) thinking about somebody/something" (Duden, 2002, p. 978). Oblivion is connected to loss, in the context of which the disappearing of something existent is both generally understood and connected to being incapable of keeping it. Thus, it includes an interpretation offer concerning the not process of oblivion, which cannot be experienced, and the possibility of forgetting a subject by leaving it out of consideration. In this context, it stays open if this "no-longer-having-in-mind" happens purposefully or unconsciously. A look at an etymological dictionary may help with opening up the dimension of meaning even further. For example, the German root word can be pursued as far as the Nordic word geta, "to achieve, to obtain". The prefix "for" reverses the meaning of a verb, so that Dutch fergeten, English "forget" - "get" can as well mean "obtain" or "receive" and German vergessen refer to the negation of "getting something" (Duden, 1989).

The etymologic analysis reveals the close connection of "to forget" and "to lose". However, the words cannot be used as synonyms, for obviously losing something precedes forgetting it. Only in the case of "intellectual property", the two terms seem to mean similar things. ${ }^{36}$ In this case, however, the problem is more complex. Whereas in the case of a forgotten key, one may go back and fetch it, the case of lost knowledge - at least from the subject's point of view - raises the question if it is possible to remember a complex intellectual content that is identical with that of an earlier time. Applied to "knowledge", oblivion increases the loss: once something is gone, it cannot be retrieved in its past state. If the definition of a term has to do with cognition or knowledge, the forgotten object is also concerned. In contrast to the forgotten key, which is available again with the whole range of its functions after being fetched, the connected cognition or the subjective meaning of a key is different. The loss described by "oblivion" must be structured according to levels of possible reconstruction, in the context of which oblivion includes both aspects of total loss and those of "re-"coverability.

Apart from such an analysis of the meaning of a word, other facets can be identified, indicating different readings of the term in everyday language. For example, there is a difference between the process of forgetting and its result. ${ }^{37}$

36 The Deutsches Wörterbuch [German Dictionary] by Jacob and Wilhelm Grimm (1956, p. 415) provides that "since its earliest appearance in the Germanic language, vergessen [to forget] can only be found referring to an intellectual activity, thus it refers to unintenionally losing something out of one's mind.".

37 In the Deutsches Wörterbuch [German Dictionary] by Jacob and Wilhelm Grimm (1956, p. 422) we find a similar distinction. However, the process of vergessen [to forget] is called an action, and the result of this action is called a state or Vergessenheit [forgottenness]. 
In the former case, it is about if and how one may lose something one used to know, and in the latter case, there is some knowledge that is currently not available.

The process of losing knowledge cannot be experienced as such - we cannot watch ourselves while forgetting. Although this way of losing something can hardly be intended, the knowledge of having lost something develops from a process of becoming or making aware. If something, such as an appointment, has been forgotten, one does not refer to a process but a current deficit. The moment one becomes aware of having forgotten something, one may ask oneself how this was possible; one may thus draw one's attention to the underlying process. Thus, common thinking at first refers to the forgotten object - no matter if it is a physical object or a cognition - to then, and only in exceptional cases, deal with reconstructing the circumstances.

Usually, oblivion is negatively connoted. Furthermore, everyday language provides the possibility to distinguish between the tendency of forgetting something and knowing about the fact that oblivion is possible. Whereas in the case of a knowledge bearer, the tendency of forgetting something may as well be described by the term obliviousness, the forgottenness of a knowledge content describes, in the context of a forgotten object, the state of forgottenness. In this sense, Friedrich Georg Jünger defines forgottenness as a precondition for oblivion:

The nature of forgottenness, however, is that [...] our forgetting "something" becomes part of it and is capable of becoming part of it. Without forgottenness there would be no oblivion and no memory. By considering that everything imagined has been, is and will be taken charge of by forgottenness, we consider the "incredibility" of forgottenness. (Jünger, 1957, pp. 12-13)

Another distinction concerns "organising" oblivion by the knowledge bearer and separates purposefully controlled from unconscious oblivion. This is a metaphorical extension of the concept of oblivion towards other fields of not-knowing. As the process of forgetting cannot be subjectively experienced, we must assume that it does not happen consciously in the case of oblivion. Nevertheless, there are manifold ways of telling people to forget something. Volitional forgetting is connected to giving up on something - object or knowledge content. For example, the phrase that we may forget about something refers to denying the concerned object its relevance for a specific social context. The demand must be understood as a call for ignorance.

Also, connecting "to forgive" and "to forget" refers to consciously giving up on knowledge content. Settling the conflict about guilt and injustice is supposed to come with forgetting, consisting of also preventing the memory of a 
bad past, apart from the unregulated attribution of guilt. By withdrawing the process of forgetting from the individual consciousness, which is incapable of intentional oblivion, and by transferring it to the longer processes of social change, it becomes politically controllable via reconcilement and through tabooing strategies and silencing.

The here presented stocktaking of "oblivion" in contexts of everyday life and the written language shall be concluded by another cursory look at an older encyclopaedia. Also, we find hints at dimensions of meaning there. Zedler offers the following characterisation of oblivion:

We have forgotten something if either we are no longer capable of imagining it at all, although in the past we used to recognize it: or at least we do not recognize it when encountering it again, or if other people remind us to the fact that in the past we used to recognize it. We will soon forget something again if either we are careless about it or if we do not often think about it, or also if the mind is occupied by this in one moment and by another thing in the other. [...] Thus, oblivion is typical of the old aged, either if they are too busy with other things or if, because of carelessness because their minds are weak, they are no longer capable of thinking about this or that they used to deal with in the past. (Zedler, 1746 , p. 666$)^{38}$

This older definition includes further aspects, some of which have not yet been dealt with. For example, the problem of attributing meaning is emphasized, in the context of which, firstly, the loss of intellectual property refers to facts. Secondly, there is an authority, which informs the forgetting person that he or she forgets something - this may refer to both traces and fellow humans. Once again, preconditions or circumstances of oblivion are mentioned: forgetfulness due to old age, overstimulation, distraction, inattentiveness, or weak apprehension. There is obviously a social problem at the heart of things in the context of dealing with individual consciousness. To sum up, oblivion refers to the fact that the observed behaviour of consciousness is different from the expected normal state based on an context of experience or stock of knowledge. Interpretations of these differences more or less address the field of the pathological - obviously, the social group provides itself with explanations for why somebody unintentionally behaves differently, although he or she should know better.

After an overview of the term's usage in everyday language, after a look at encyclopaedias, and after having reconstructed three dimensions of the

38 This is only a short excerpt from a longer section in which Zedler's encyclopaedia (1746, p. 666) sheds light on the phenomenon of oblivion in a number of different action fields such as religion, law or medicine. 
common use of language, the search for further aspects of meaning will be continued within the stock of cultural tradition.

\subsubsection{Oblivion Within the Circle of Life}

Written documents discussing the issue of oblivion are already found in texts on Greek mythology. Lethe, the goddess of oblivion, grows from the race of the night. She is the counterpart to Mnemosyne, the mother of the muses and goddess of memory. Lethe is furthermore one of the rivers of the underworld. It provides the deceased during their transmigration of souls with oblivion, liberating them from the memories of their lives and preparing them for being reborn in a new body (Weinrich, 2004). It is this figure of thought which provides the background for Plato's considerations on recollection.

For Plato, whose thought is based on the idea of the transmigration of souls, human cognitive faculty is based on universal ideas. However, this universal knowledge is forgotten at birth and can be recollected - we might as well say: learned - in the course of a life. ${ }^{39}$ However, this is not complete oblivion, as otherwise, it would be impossible to have ideas or be pointed to them (Weinrich, 2004, pp. 19-20). To illustrate this figure of thought, Plato refers to the example of the wax tablet. As papyrus was expensive, one used wax tablets for everyday notices, into which one could carve or "impress" everything one wanted to remember for a short time. Thus, the wax tablet was a recording possibility with an oblivion function, as after using it, it was smoothed again and could then be reused, overwritten. According to Plato, also the human soul must be understood to be a wax tablet. At the time of his/her birth, any person is a "blank paper" (tabula rasa) which is written on or impressed throughout his/her life (Weinrich, 2004, pp. 20-21). True knowledge is created if impressions coming from sensual perception are congruent with the ideas of higher reality. ${ }^{40}$

39 In Plato, this aspect of the ancient oblivion doctrine is found in the the Menon dialogue. It is Plato's protagonist, Sokrates, who provides evidence for humans being incapable of inventing new things and only being capable of recalling already existing ideas. With the help of skillful questions, Sokrates makes a young boy who is not familiar with mathematics aware of how to calculate the area of a square. The theory of recalling is further elaborated in the Phaidon dialogue, also the classical insight according to which oblivion as the starting point for insight is to be found there (see Kreuzer, 2010).

40 The idea of equality is inherent to the souls: sensual perception may be referred to ideal models one has seen not in lifetime but before birth (see Yates, 1999, pp. 36-37). This figure may be understood to be a predecessor of the scheme theory of psychology, although the latter does not provide for a transmigration of souls. In Plato's Theaetetus dialogue (Plato, 1921) this is given as the reason for the difference between knowledge and opinion. Whereas true knowledge consists of perception content and idea being congruent, 
Plato imagines the capability of memory, which he believes to be necessary for rhetoric art - in the sense of leading the soul - as consisting of three parts: firstly, this performance of the consciousness consists of the mental storing of data (hypomnesis), secondly of the copy function (mneme), and thirdly of remembering the non-identical (anamnesis).$^{41}$ With the help of this distinction of different concepts of memory, he demonstrates that the idea of a memory that functions as a data store is absolutely insufficient: if impressions could simply be conserved and stored, false memory would be ruled out. However, the memory is no mental container from which data stored through the act of remembering could be retrieved. The possibility of a copy that is absolutely congruent with the original event is thus ruled out. Remembrance is a constructive process.

Closely connected to the remembrance theory formulated in contrast to all-comprehensive oblivion due to birth and death are the considerations on literacy. In an allegory, Plato uses the ambiguous word phármakon, which means an aid on the one hand, but poison on the other. Writing as an aid for storing memory contents relieves memory; however, it entices us to only rely on this invention and neglect remembrance entirely. Plato states that due to the possibility of fixing something in writing, the souls are fed with oblivion. Furthermore, writing could never replace remembrance (Kreuzer, 2010), as it does not make a copy of the thought which is stored in it; once again, reading requires memory. In this context, reading is always recognition - if not of information, then at least of the structure of meaning and semantics.

Finally, another figure of Plato's memory theory is the insight that the process of remembrance cannot be equated with its subject. That what is impressed into the wax tablet consists of impressions but not of events. Aristotle, who while giving up on the idea of the transmigration of souls - connects to Plato's considerations, later states that what can be remembered is always an image of something but not its impression or imprint nor a copy of the original. Now remembrance presents itself as the presence of the non-present; it refers to things past. This symbolising and symbol-understanding faculty distinguishes humans from animals (Kreuzer, 2010, pp. 263-264). Whereas Plato established a connection between oblivion and death or birth, with Aristotle at the latest, the focus must have shifted on forgetting the original sensual perception

opinion is a perception content without such a comparison. In this context it may be that the comparison is not based on an idea but just on a perception made present - the result is a wrong opinion (see Janssen, 2001).

41 Plato has Sokrates explain this in the Phaidros dialogue. 
in the course of imprinting and remembering, in the course of producing a reconstruction.

In Aristotle, however, oblivion is hardly discussed in detail; it becomes much more important for the considerations by the Aristotelian Plotinus. There, remembrance must be equated with storing, which results in piling up a vast stock of memory contents. The soul - consciousness - is interested in escaping, via oblivion, this manifoldness into amnesia, a state of having no memories, into simplicity. Remembrance, which creates variety and difference, temporalizes thinking. Plotinus finally connects it to the temporal dimension of the past. Then oblivion contrasts remembrance as an "ecstatic unification beyond the temporal" (Kreuzer, 2010, p. 264). Johannes Kreuzer demonstrates that Plotinus formulates a figure of thought which conceives remembrance as the "method and tool of intellect taking-the-inward-turn"; however, oblivion is conceived as the "negation of all remembrance", which is preferred over remembrance. Only oblivion allows the mind to leave the sphere of the finite behind (Kreuzer, 2010, p. 265).

Oblivion, which is positively addressed in Plotinus and helps the consciousness defending against too many memories by striving to go back to the simplicity of the modesty of thought, may be connected to current ideas of the tendency towards complexity reduction and also to the question of the (social) consequences of compiling too much knowledge.

A philosophy-historically influential continuation of the theory of remembrance and oblivion as started in Greek antiquity is found in the work of church teacher Aurelius Augustinus who, in the Tenth Book of his autobiographical confessions, unfolds a theory of memory which is based on Plato and Aristotle. In the first part of Confessiones, Augustine reflects on his life as a pagan and sinner. Why finally he found his way to the Christian faith is an unanswered question for him which - by reaching back to the doctrines of mnemotechnics he was familiar with as a former teacher of rhetoric (Yates, 1999, pp. 46-50) - then leads him a subject-centred philosophical-psychological theory of memory.

Based on ideas of the transmigration of souls and recollection, Augustine replaces Plato's doctrine of forms - according to which every insight is already inherent to the soul and was only forgotten at birth - by the insight that God as the creator of the world has His place in human memory. ${ }^{42}$ However, the humans have forgotten this closeness to the Divine, which is why God sends

42 Here Augustine develops a complicated conclusion: as God cannot be encountered at any topographical place - and certainly not in the form of an image - and is thus immaterial, also memory must be immaterial. "The discovery of something that is nowhere is, for Augustine, the realization of the reality of immaterial being: an adaptation of Neoplatonic method is again evident. If God is everywhere and nowhere [...], and ideas are nowhere $[\ldots]$, then both are immaterial, if distinct" (O'Daly, 1993, p. 32). 
out signals to them and lays traces to lead them to the sometimes windy paths leading (back) to Him. Augustine describes the road to conversion and the confession of God as a remembrance. In other words: the search for God which was also the search of the author of Confessiones - consists of looking for indications of God in the forlornness of "godforsakenness" (oblivio dei), to finally join the "community of memory of Christian belief" (Weinrich, 2004, p. 22).

Thus, there is a connection to the insight that sinful oblivion is not the antithesis to remembrance but memory. Oblivion is never total - the memory always triggers remembrance because something has been forgotten. Thus seen, oblivion is the precondition for remembrance, for only if and because something has fallen into oblivion, there is the necessity of reflecting consideration. To put it in a nutshell: stimulating memory as well as the inward attempt at a reconstruction of what has been forgotten is what triggers and makes memory.

On the other hand, also the process of remembrance has got nothing to do with storing contents in the memory. Neither is something preserved nor is it imaginable that something which once has been remembered could not be forgotten again. If everything remembered was stored, this would be a traumatic limitation in the sense of not-being-able-to forget. Thus, remembrance is a permanent process, happening because of constant oblivion over time. Moreover, as the consciousness is not timeless, also the process of remembrance is part of memory. Thus, as remembrance is omnipresent and infinite, the remembering subject cannot be an a priori shaped, given and stable I, but it constantly creates and changes its identity by combining references to chronologically different events.

In his work on Trinity, Augustine develops his theory of memory further. Although there is hardly any emphasis on oblivion, he works out influential models for later philosophising on memory, remembrance, and oblivion issues. Based on the Divine Trinity of Godfather, Son, and Holy Spirit, he identifies a parallel to the mental faculties of memory (memoria), reason (intelligentia) and will (voluntas). If these two trinities are placed next to each other, a connection between Godfather and memory becomes obvious. The Creator God created the world. In His memory, all the blind spots coming from sinful human oblivion are included (Weinrich, 2004). Remembrance comes from the interplay of all three mental faculties. Thus, it has nothing to do with just activating or obtaining some data stored somewhere, but it follows reason and, most of all, will. ${ }^{43}$ In other words: willingly one recognizes an internal process, and apart from being oriented at certain topics or subjects, this process refers

Here as well as in the following, see Johann Kreuzer (2010). 
to itself again. By referring to something else, memory always also refers to itself.

As remembrance happens constantly, it may be assumed that not every process of remembering is consciously reflected on. Much happens by so-called minor memories, producing the effect that humans have much more things in mind than they are currently aware of. Accordingly, Augustine understands thinking to be self-remembrance, the will and desire to update that what has already been laid out in the depths of consciousness to become insight.

However, if remembrance is understood this way, the consequence is that remembrance cannot exclusively refer to things past. As it is a mental process always happening only in the here and now, remembrance may refer to the past, the present and the future at the same time. This results in the complicated construction that the present is always already remembered. Everything is grasped by the consciousness in the present moment and only refers to memoria, intelligentia and voluntas. Remembrance as a cognitive process always happens primarily and must not be understood as a secondary process in the sense of "considering-once-again" (Kreuzer, 2010). In other words: Augustine's theory of time communicates the insight that there is no objective difference between past, present and future but that there is only the present which is related to past and future. The experience of time results from the consciousness switching between these categories of time (Saar, 2001). Thus, also the process of oblivion always happens in the present. Then, through the problem of time, irritation caused by traces, for example, allows for becoming aware of both oblivion and what has been forgotten.

\subsubsection{The "Nature" of Oblivion}

If we start from the knowledge bearer, for an interpretation of oblivion, we will first turn to the loss of knowledge of the individual. It is then about "natural" or "automatic" oblivion. The basic assumption, which was already Augustine's, is that any consciousness constantly forgets. This may be attributed to the nature of man, or it may be due to the consciousness being dependent on its environment and the latter's continuity. This is in line with the Augustinian motif of any remembrance referring to remembrance while at the same time influencing the current perceptive faculty. Consciousness is thus a chronological process with a permanent share of remembrance - everything beyond this communication process between subjective present and past is forgotten. Thus, an aspect of human nature would be forgotten, which could be controlled. Accordingly, the initial problem for a broad tradition of memory and remembrance techniques is that humans cultivate their nature and construe their history to learn from it. 
Apart from the neuro-scientifically explainable problem of oblivion and the research of ways of making use of this knowledge both in the sense of avoiding an unwanted loss of knowledge and purposefully preventing unwanted remembrance, in the field of allegedly "natural" oblivion aspects must be taken into consideration which may be called culture-induced oblivion. Ways of losing knowledge, which evade everyday reflection and are thus a subject of scientific analysis, are both in the "nature" of the brain and the "nature" of the social or the cultural.

If sociological study deals with a subject on which other disciplines have already presented research results, these should be appreciated because, firstly, the semantics sociology must reach back to have been influenced by this research tradition. Secondly, it is obvious - most of all concerning knowledge, remembrance, and oblivion - to reach back to the subject's consciousness, even more as, after all, the individual must be considered a knowledge bearer also because of the collective consciousnesses it belongs to.

When searching for answers to memory or oblivion, psychology and brain research are prime addresses, also from a sociological point of view. Whereas neurophysiology is about exploring the brain's biochemical way of functioning, in the context of which issues of memory and oblivion are addressed, psychology is about gaining insight into thought operations and thus about reaching back to and the effect of experiences on current behaviour. Despite these differences, particularly when it comes to methods, the two research disciplines have always been mutually dependent. For example, young Sigmund Freud oriented himself at the neuro-physiological insights of his time, ${ }^{44}$ and neuro-biologist Eric Kandel explains his research motivation by his desire to biologically substantiate the insights gained by Freud's psychoanalysis (Kandel, 2006). It is remarkable in this context that, when it comes to finding explanations for the ways of functioning of memory or oblivion, one always starts from the thus connected problems. One main interest of brain research is the analysis of brain damages; psychology focuses on the analysis of malfunctions of the consciousness - in this case, one frequently starts from what in everyday language is meant by the term oblivion. ${ }^{45}$

\footnotetext{
44 See the encyclopaedia entry on Freud by Birgit Boothe and Jürgen Straub (2001).

45 Daniel L. Schacter (2001) calls the failures of the brain the Seven sins: Transience refers to the memory becoming weaker over the course of time, absence of mind refers to a weakness when it comes to information being stored by the memory, and blockade refers to the difficulty of recalling existing information. Apart from these three sins of omission, Schacter identifies four sins of commission: in the case of misattribution a memory is attributed to a wrong source. Suggestibility refers to the instability of memories as a result of influences. Distortion happens if memories of past events are reinterpreted against the
} 
Nevertheless, these research disciplines generate several terms for memory, some of which, such as the distinction between ultra-short-term, short-term and long-term memory, refer immediately to the issue of oblivion: how long and under which circumstances is perception content or information stored?

Both brain research and nomological psychology started at the end of the 19th century. The following will not be about chronologically sketching the history of these two research disciplines - with a particular focus on oblivion. Instead, light shall be shed on essential concepts and theory offers of neuroscientific oblivion research to develop the stock of terms by help of which it is possible to understand or explain the process of oblivion. The first of these approaches are the studies by psychologists Ebbinghaus and Bartlett, which must be called classical works. Then essential aspects of the amnesia concept of brain research will be presented.

Many consider the study by learning researcher Hermann Ebbinghaus a very early work experimentally dealing, from a psychological point of view, with the issue of oblivion (Ebbinghaus, 1998). He consolidates the state of research of the psychology of his time by three positions. The first one consists of the assumption that each perception leaves an impression, a trace, which is always weaker than the original perception. These memory images are eclipsed by newly arriving perceptions. However, in the dream, when perceptions coming from the environment are lacking, recent memory images are sometimes very present. Older images are frequently eclipsed by newly arriving ones. Once these layers have been broken up or pushed aside, the memories stored there appear again in the original quality. The second position assumes that with growing chronological distance, memory images become ever darker, lose conscience intensity and are replaced by more recent images. However, these slowly sinking images cannot be imagined as a permanent yet qualitatively minor stock of memories but as a starting point for possibly recreating the slowly fading imagination contents - thus, it may be that the "apparently forgotten idea arises again in perfect clearness" (Ebbinghaus, 1998, p. 64).

The third concept of oblivion which was common in those days is based on the opinion that memory contents slowly crumble - over time, memories become ever more incomplete, and increasingly the links between them are lacking. In this context, the problem arises that possible mental links between memory fragments become equally probable and that, when remembering, one must decide about what things have probably been like. Ebbinghaus assumes that all three variants of explaining the process of oblivion are only

background of current knowledge, and persistence addresses the problem of simply being unable to forget certain things, a problem which is also relevant for trauma therapy. 
partly true, as each of them suggests just one valid interpretation of the subject of memory. It cannot be taken for granted that it is possible to find out how many and which memory elements are still there or which degree of darkness has been achieved in the given moment.

Ebbinghaus is not first of all interested in formulating an oblivion theory. Rather he wanted to find out according to which laws the brain functions when we try to keep something in mind in the long run by repeatedly memorising something. However, he succeeded in finding a formula also for "natural" forgetfulness. His self-experiment consisted of creating many systematically construed combinations of syllables that did not make any sense to the observer. He learned them at heart, by again and again reading and then speaking individual rows of syllables. This procedure was repeated at regular intervals, and the efforts made during memorising were modified. At first, he found out that in the beginning, oblivion happens very fast and then more slowly. ${ }^{46}$ Thus, in the beginning, the oblivion curve rises steeply, to then become even flatter. Thereby, it can be demonstrated that complete oblivion happens only after a very long time.

This way of proceeding - learning abstract rows of syllables - was met with strong resistance by British psychologist Frederic C. Bartlett. After applying Ebbinghaus's method, he found out that the memory does not work exclusively reproductively but actively construes memories. Oblivion, he stated, does not happen as a decay progressing over time; instead, remembrance happens depending on the situation and creatively reaching back to existing experiences. Memory is meaningfully structured, and remembrance happens primarily by creating, updating and changing mental schemes. In his experiments, Bartlett did not make the test persons learn nonsensical rows of syllables. Instead, he presented them with stories coming from cultural spheres which were alien to them. Then, one test person had to tell the respective story to another one and so on. As a result, Bartlett not only found out that the story became ever shorter, but he also discovered that it was more and more adjusted to the narrators' cultural sphere - less familiar aspects and those the narrator believed to be unimportant were left out. Obviously, the narrators remembered most of all aspects they were familiar with, and thus those elements of the respective story could refer to schemes that were available for

46 Ebbinghaus describes the formula according to which the oblivion function works as follows: "the quotients of the amounts retained and the amounts forgotten are inversely as the logarithm of times" (Ebbinghaus, 1998, p. 78). Time is measured in minutes, starting with one minute after finishing a learning phase. 
them. ${ }^{47}$ Schemes are permanent knowledge structures that serve for organising experiences and may be understood as starting points for both remembrance and oblivion.

In brain research, amnesia refers to the phenomenon of the loss of memory, the emphasis being on abnormal memory disorders. Like neuro-physiologicalpsychological concepts of memory, also the field of amnesia knows several differentiations. One crucial distinction refers to the time axis of memory. The remembering access may address the past, the remote memory, or the shortterm memory, which refers to the future. If there is damage to the remote memory, one speaks of retrograde amnesia. It concerns contents that were coded before the disorder - and this means: changed into storable information $^{48}$ - and the access to which is now disturbed. An injury of the brain, on the other hand, may affect the recording of newly arriving perception contents. Accordingly, one speaks of anterograde amnesia if the formation of the short-term memory is affected.

Some of these different kinds of amnesia can be located in certain areas of the brain; however, they are commonly attributed to certain kinds of memory, in the context of which the memory is not understood as a place in the brain but according to its memory function. The latter can be differentiated according to conscious, communicable contents or routine, "automatised" and exercised knowledge one is not aware of during everyday use. Conscious knowledge is attributed to declarative memory; knowledge incorporated in a learning process is found with procedural or implicit memory (see Roth, 1996). Declarative memory can now be further differentiated into semantic and episodic memory, in the context of which the latter is understood to be a "decoration" of the former which has genuinely developed in the course of human

47 On this see the short portrait of Bartlett by Carlos Kölbl and Jürgen Straub (2001a), who elsewhere also offer a definition of the scheme concept as a "comparatively stable knowledge structure which is resistant against oblivion processes [...] and guides perceptions while allowing for cognitively integrating known and unknown facts, the expected and the unexpected" (Kölbl \& Straub, 2001b, p. 520). The study Remembering. A study in experimental and social psychology, in which Bartlett (1932) introduced the scheme concept into memory research, was published for the first time in the same year as the treatise on the Phenomenology of the Social World by Alfred Schütz where a similar scheme concept was defined: "A scheme of our experience is meaning-context which is a configuration of our past experiences embracing conceptually the experiential objects to be found in the latter but not the process by they were constituted" (Schütz, 1967, p. 82).

48 Storable information is also called an engram or memory trace. The coding as an engram may also be described as being filed by the short-term memory. Storing in the long-term memory happens only if the engram is connected to other engrams which have been coded earlier. This process is called consolidation (see Piefke \& Markowitsch, 2010). 
evolution..$^{49}$ Only episodic memory allows humans to have mental access to their past. Indeed, also animals are capable of acquiring knowledge that is independent of themselves and of time. However, no awareness of the self in subjective time can be determined.

The neuro-biological research of the first heuristic concept of episodic memory is based on distinguishing two kinds of amnesia: temporal amnesia refers to a "loss of the memory of personal experiences", categorial amnesia, on the other hand, refers to a loss of "the memory of learned facts" (Tulving, 2006, p. 6o). Both kinds of loss of memory have been proven in the context of brain damages, but not always does obvious damage of the brain result in amnesia. On the one hand, it may be that the memory is intact despite damage to the brain area. On the other hand, it may be that there happens a loss of memory although the brain is undamaged; consequently, the latter cannot be identified with the help of neuro-physiological examination methods. Such disturbances of memory may appear e. g. as a result of traumatic experiences; these disorders are called psychogenic amnesia (Piefke \& Markowitsch, 2010). There is also the insight that amnesia may be caused by access being blocked due to stress when the release of stress hormones results in nerve cells being occupied. Then the occupied cells are no longer capable of absorbing newly arriving transmitter substances. Information is not only processed at one place in the brain - there is not just one nerve cell for the picture of grandma - but connects to many areas; in such cases, we may not assume a total blockade. Rather, one recognizes a kind of selective perception that would happen differently under different circumstances. Thus, the respective information and a complete network of connected pieces of information constantly change its shape, particularly if it is about complex biographic-episodic issues such as love.

If, however, such a structure is disconnected at one place, one speaks of the disconnection syndrome. Then the individual does no longer succeed with "generating the access to information or [with] recalling memories which allow for connecting newly acquired and already existing information to each other ('association')" (Markowitsch, 2001, pp. 238-239). If information cannot be newly inscribed, the memory is blocked or suppressed. This may then be experienced as oblivion when wondering why the so hurriedly and intensively "drilled" information is so poorly kept in mind. What is confusing is that this

49 The concept of episodic memory was essentially developed by Endel Tulving. The here presented information comes from a short overview of the history of the concept (Tulving, 2006, p. 57). 
is no problem of access to information (retrograde amnesia) but a problem of storing information (anterograde amnesia).

Sigmund Freud dealt with oblivion on a large scale in his work Psychopathology of Everyday Life of 1901. In the course of the individual chapters, he analyses how it is possible that in the course of a conversation, one frequently forgets personal names, words in foreign languages, or combinations of words as well as intents, that again and gain one remembers an event which does not seem to be particularly worthy of being remembered. ${ }^{50}$ Freud is not ready to accept that the only reason could be insufficient knowledge of the respective term. Instead, he attempts to derive oblivion from the context of the conversation and concludes that, apart from simply forgetting things, oblivion may also be motivated by suppression.

Suppression is a crucial concept of psychoanalysis. It refers to a mental process in which the access to coping with affect-laden, emotional desires or aspirations is prevented because they are kept in the unconscious. However, as the thus connected emotions strive for finding expression, they break out by way of being converted into somatic phenomena (Freud, 1949, pp. 128-129). In other words: suppression describes a state in which the consciousness, but not the unconscious, has forgotten a drive. As this drive can neither be consciously controlled nor successfully suppressed, it takes another road towards "articulation". Such a "jailbreak" may happen in the form of mental illnesses or anomalies, but it may as well find expression as a minor suppression of moments of displeasure in everyday language, by way of Freudian slips or indeed by way of "coincidentally" forgetting terms or names.

Thus, oblivion which is not controlled by the consciousness, becomes an indicator of mental illnesses and justifiable displeasures concerning past biographic events. Accordingly, Harald Weinrich states:

With Freud forgetting loses its innocence. From then on, anyone who has forgotten something or wants to forget something has had to defend himself and face the possibly painful and embarrassing question Why? The more firmly he is convinced that his forgetting requires no justification and that he has simply forgotten. (Weinrich, 2004, p. 134)

Oblivion must thus be understood as being, if not intentional, then at least functional. In case of such an attribution, the decision to forget something may be supposed to usually not be intentional, intending to forget moments

50 So-called screen-memory is a substitute memory - what is forgotten is other, more important impressions which, for reasons of infant amnesia or traumatic experiences, are hidden behind memories which seem to be trivial (Freud 1901). 
of displeasure. It results from the confrontation with an uncovered oblivion event: "Why did you forget this?". This question compels to a retrospective attribution of meaning - to the construction of a reason for this "blackout".51

By reaching back to the Freud disciple Pierre Bertrand, Weinrich asks about the value of oblivion for psychoanalytical therapy (Weinrich, 2004). At least, specific kinds of traumatic memory are evoked in the context of psychoanalytical treatment. This allows for recognizing and lifting a suppressed trauma as well as the neurotic disorder resulting from it due to sublimation. However, now the patient is provided with new, possibly burdening knowledge. How to deal with this new knowledge, if he or she is supposed to keep it in mind or forget it, is not answered by Freud. It is assumed that it is possible to live an ordinary life after successful therapy and that the cause for renewed suppression and sublimation has disappeared.

The method of psychoanalysis aims at the treatment of psychoses and neuroses, which are said to be due to suppressed drives or traumatisation. It follows the remembering-repeating-coping-with principle (see Freud, 1914), the uncovering of the cause of the disorder and the then following coping with the disorder itself happening in the mode of rationalisation. Thus, it is about forgotten things that are supposed to be remembered again, to then again forget everything - that is, traumatisation, neurosis and therapy - if necessary. ${ }^{52}$

In view of therapy for mental disorder appearing due to traumatising experiences - posttraumatic stress disorder, e. g. after war or catastrophes - two basic orientations become apparent. Whereas psychological approaches - such as in the context of behavioural therapy - aim at the treatment of pathologic symptoms, psychiatric research attempts ways that start from remembering traumatic events and aim at undoing the causes for a disorder with the help of pharmacologic therapy. The focus in this context is on the development of medication with the help of which a traumatising memory can be purposefully deleted from the memory. Whereas for the time being, such a punctual therapy is still only found in science fiction literature, medication is already

$5^{1}$ On this see the german title of Daniel L. Schacter's book on the Seven Sins from 2005: Aussetzer - wie wir uns erinnern und vergessen [Blackouts. How we forget and remember].

$5^{2}$ Already at an early stage, Theodor Reik (1920) extended the suppression concept on collectives. Under the title Über kollektives Vergessen [About collectice oblivion] he extends the concept of individual oblivion which Freud had developed in his psychopathology of everyday life, assuming a cognitive failure caused by suppression. Reik's considerations start out from watching a group of young adults talking about a book whose title none of them remembers - although all of them have read it. From the psychoanalytical reconstruction of the case there concludes that there exist "forbidden thoughts" and topics which, dependent on each respective culture, are taboo and, by being associated with words or elements of words, inhibit speaking or even remembering certain terms. 
used, which is capable of not blocking the memory but indeed a specific kind of neuronal nodes. For example, medication use allows for preventing incoming information from docking with those nerve cells as being in charge of emotional processing. For instance, the Propranolol beta-blocker reduces - similar to alcohol but without the thus connected changes of consciousness - emotional perception as a whole. Thus, taking this medication before an expected traumatising event reduces the later development of a posttraumatic stress disorder. However, it may also be retroactively used for trauma therapy. As any reaction by the consciousness to a trigger stimulation connected to a traumatising experience causes a new memory of fear, it is possible to inhibit the connection of neuronal stimulation and emotion with these "oblivion pills". Consequently, the fear connected to trigger stimulation and traumatic memory ceases; now, the experience can be reflected "soberly" and perhaps even be forgotten entirely (see Brunet et al., 2007).

Already the availability and use of a means with the help of which the damage risk connected to probably traumatising events can be reduced may be understood as making instrumental use of oblivion. For the time being, possible effects on society's way of dealing with traumatisation-relevant hazards as far as to the everyday, mass use of legal oblivion drugs and the resulting processes of cultural change are a subject of controversy. ${ }^{53}$

Another approach is provided by the psychological research of guided or controlled oblivion. Studies on directed forgetting focus on the experimentally analysed question about an improvement or deterioration of memory if lists of words are learned, sometimes - or sometimes not - connected to the demand to memorise or forget them. With the basic form of the experiment concerning the short-term memory research, the condition for directed forgetting is that the number of words to be memorised and to be forgotten is the same - twelve each. Now, this condition is contrasted to two logical examinations - a list of exclusively twelve and a list of 24 words to be memorised. Under the condition that also words to be forgotten are marked, the test person is told that later only those words will be examined he or she is supposed to memorise. The directed-forgetting effect measures how well the test persons remember the twelve "critical" words if they are confronted with different test conditions only twelve words to be memorised, twelve words to be memorised, and twelve

53 This was already discussed at an early stage, in the report by the President's Council on Bioethics (2003) initiated by US President George W. Bush - for a sociological point of view see Christoph Lau, Peter Wehling and Oliver Dimbath (2011). 
words to be ignored, 24 words to be memorised etc. ${ }^{54}$ It turns out that words that were supposed to be forgotten are more frequently forgotten than those supposed to be memorised. Information, we might generalise, which is declared unimportant, is indeed not stored for future remembrance.

From these spotlights on intended oblivion, we may conclude that the loss of knowledge may be connected to the neuronal organisation of reaching back to "imprints" and to external control of absorbing information. Both psychoanalytical and psychologic approaches seem to be based on the assumption that the brain guarantees practical capacity to act. Knowledge that seems to be an obstacle for coping with current situations is suppressed or simply not recalled, whereas, in the case of disorders, this system may get out of step. Accordingly, it may be that important information is blocked due to stress or that unwanted, obstructive information is an obstacle to an adequate course of action. However, these approaches are different from each other concerning whether unused knowledge really disappears or if it still influences the operations of the brain. From these differences, there derive the various strategies of using the brain's way of working if things are supposed to be purposefully forgotten.

If we do not understand the individual as the bearer of knowledge but the social group and the culture it unfolds, we also state aspects of forgetfulness. These are only partly derived from a metaphorical transfer of the oblivion concept from the psychic to the social. In this context, Aleida Assmann speaks, among others, of "automatic oblivion", 55 which may be said to be due to disappearance in the material, biological or technical sense. In the context of the presented overview, some kinds of socio-cultural forgetfulness that are usually not in the focus of everyday understanding will be shortly presented.

One kind of social forgetfulness is the decline of knowledge due to changing meaning structures or semantics of society. The ethnologist Julian. A. Barnes unfolds the concept of structural amnesia in the context of family relations and thus connected: entitlements - in tribal societies. ${ }^{56}$ In contrast to a written

54 A comprehensive overview of different aspects of directed forgetting is presented by Colin M. MacLeod (1998).

55 Also Aleida Assmann (2012) calls oblivion the "normal case" in culture and society, in contrast to which remembrance is connected to efforts. Even objects which have been carefully kept by individuals are destroyed or distributed after these individuals have deceased and are attributed with new meanings in new contexts. Automatic oblivion happens in the course of material disposal as well as according to the biorhythm of the generations in the course of which the experiences of the older generation are at regular intervals devalued and replaced.

56 In Paul Connerton (2008), structural amnesia according to Barnes constitutes the fourth type of oblivion. 
fixation of kinship structures, he reports that ancestry is proven on the village square by presenting witnesses. It is remarkable that in this context, some inhabitants do not appear under family relations. Structural oblivion as a forgetfulness principle is based on the fact that members of a community remember only those relations which are relevant for them. This becomes structural, for example, in the case of sharing names. According to the studies quoted by Barnes, those ancestors are still remembered who shared their names with certain families. On the other hand, in British society, which functions as a comparison, the inheritance of names happens patrilineally, in which female names are forgotten. Whereas a Maori male can tell up to twenty generations of his ancestors to legitimate his status, in societies where ancestry is of little relevance, the kinship structures are most of all forgotten or only rudimentarily remembered.

Combined with a high degree of geographical mobility, the state of affairs may be reached that is typified by the possibly apocryphical story of the American living in Denver, born in St. Louis, whose Mother was born somewhere in the Eastern States, whose father was born somewhere in Europe, and who doubted if he ever had any grandparents. (Barnes, 1947, p. 53)

Forgetfulness, this we may conclude from Barnes's study, is closely connected to social relevance structures: what is unimportant or may become unimportant is not remembered. Jack Goody and Ian Watt take up the concept of structural amnesia and find that organisational change - we might say: social change - influences structural forgetfulness.

They can do this more constantly because they operate within an oral rather than a written tradition and thus tend to be automatically adjusted to existing social relations as they are passed by word of mouth from one member to the society to another. The social element in remembering results in the genealogies being transmuted in the course of being transmitted; and a similar process takes place with regard to other cultural elements as well, to myths, for example, and to sacred lore in general. Deities and other supernatural agencies which have served their purpose can be quietly dropped from the contemporary pantheon; and as the society changes, myths too are forgotten, attributed to other personages, or transformed in their meaning. (Goody \& Watt, 1968, p. 33)

These dynamics of structural amnesia are slowed down only with the introduction of writing. Literacy reduces the strong relation to the present, whose relevancies determine the structure of narrations referring to the past. In illiterate society, myth and history melt into one, "[a]nd as the individuals of each generation acquire their vocabulary, their genealogies, and their myths, they 
are unaware that various words, proper names and stories have dropped out, or that others have changed their meanings or been replaced" (Goody \& Watt, 1968, p. 34).

However, structural amnesia is no exclusive feature of oral cultures. Some structural patterns are preserved even after societies have long become literate. For example, the patrilinear inheritance of names has long been preserved even in modern Western societies - and was even legally substantiated by way of written laws. Aleida Assmann even concludes on a general principle of structural amnesia and even structural remembrance from the patrilinear inheritance of names. For example, families whose names are dominated by male names come along with forgetting the female aspect of identity as far as it is connected to the family's ancestry. At the same time, the male line is remembered "automatically". In other words: women are forgotten, and men are remembered. ${ }^{57}$

Any remembering reference to past events is selective, in so far as the emphasis on one subject leaves other subjects out of consideration. Regarding the communication situation, any shared memory incorporates oblivion in so far as certain aspects are not communicated and passed on as a narration. Thus, any relation creates its own specific, narration-based identity by basing its "consciousness" only on specific aspects of personalised memory. What is irrelevant in a given narrative situation remains to be ignored and does not produce any follow-up communication. In this sense, oblivion as a result of leaving things out is not meant in the destructive sense but as part of the construction process - also when it comes to social reality. 58

Construction-related selectivity does not exclusively develop from the communication between two consciousnesses but is - following Halbwachs's framework theory - guided by social reference frames. Communication is oriented at relevant social patterns. ${ }^{59}$

The selection motif is also found in organisation research. Organisational routines are most of all understood to be aspects of organisational memory and are sometimes criticised for being obstacles to adjustment. However, some

57 Aleida Assmann (2006) continues this object-related differentiation of structural oblivion and remembrance at the level of the subject. There, by reaching back to Nietzsche, she states that women rather cultivate memories, whereas males are rather interested in forgetting.

$5^{8}$ In Paul Connerton (2008), this kind of oblivion is in line with his third type of oblivion.

59 Halbwachs' frame concept will be discussed in more detail elsewhere. Aleida Assmann (2012) files this kind of selective while at the same time culture-specific oblivion under her seven types of oblivion. 
opinions understand routines to be triggers of organisational oblivion. For example, they form a specific selection of serially combined regulations from a vast space of possible actions, thus representing a highly selective practical memory of the organisation (see Casey \& Olivera, 2011).

Oblivion, happening imperceptibly given the creation of the new, happens e. g. also in2 the reorientation of individual or collective identities. Connerton gives adopting the name of a spouse as an example. One's own original name is no longer used, and the family ancestry is made invisible. In a partner relationship, furthermore forgetting about earlier sex partners is expected. In certain kinds of families, personal identity may be oriented at the partner relationship, and when such a partnership begins, it may require renovation. "Forgetting then becomes part of the process by which newly shared memories are constructed because a new set of memories are frequently accompanied by a set of tacitly shared silences." (Connerton, 2008, p. 63). The situation is similar with professional careers - for example, moving from the worker's position to that of the foreman may result in forgetting about the problems connected to the worker's position (see e. g. Ashforth \& Meal, 1989). In the field of collective identities, such a kind of oblivion may happen if companies are taken over. Sooner or later, the members of organisations bought up by others will forget their ties to the old company's name and the connected relationship structures or make continued relationships part of the new organisational frame.

If consciousness is overwhelmed by sensual impressions, we speak of a sensory overload. Coping with such an overload consists of an increased selection of relevant information. In the field of cognition science, one then speaks of informational overload. However, a preselection happens not only through selective perception but also because of memory retention. If too much information of equal value is perceived, this may also result in oblivion. In social groups, information overload appears as soon as social acting also depends on archived knowledge and the archive's storage capacity is extended, for example, through technological optimisation. Then it may be that for decision-making processes, an amount of relevant information is provided, which can hardly be coped with. Concerning the exponential growth of scientific knowledge, we may state election automatics that - in the sense of not always intended oblivionism - come along with certain path dependencies of remembering and comprehensively forgetting about "irrelevant" information. ${ }^{60}$ Also, in Niklas

6o A hint at such a kind of oblivion is provided by Paul Connerton (2011) in a more recent edition of his essay on the seven types of oblivion. Under the "cancellation" type which, against the background of the distinction made here, must rather be attributed to culturally-intended oblivion, he shortly addresses the scientometric distribution of 
Luhmann, whose considerations are going to be discussed elsewhere, we find the motif of an oblivion function of the social system, which is supposed to prevent an overload of the system's capacity of processing information.

Another kind of social oblivion is identified by Connerton (2009) in his book How Modernity Forgets. The focus is on the assumption that change is accelerated under conditions of social modernisation and that in certain fields of the social we must expect an increasing appearance of oblivion processes. Basically, this holds for any innovation. Whenever something new is created, it replaces the old. Innovation must - also in the sense of political economist Josef Schumpeter - be understood as creative destruction. However, such creative destruction does not only happen in research and development but most of all also in the field of everyday consumption. Connerton demonstrates this with the example of modern media consumption, which, in his opinion, leads to cultural oblivion par excellence. Connecting to Walter Benjamin, he considers the daily reading of newspapers a kind of memory loss, as the thus perceived fragmentary information can no longer produce affectional experience. The focus on the new and fragmentation are increased in the case of TV consumption, in the course of which one reaches back to images and no longer to objects that can be experienced. Thus, he says, one function of the mass media is not production or consumption but making the experience of recent historical events fall into oblivion as soon as possible (Connerton, 2009, p. 84).

Then, however, Connerton has a particular focus on the topography of oblivion, which he makes plausible by the example of the development of the cities and the speeding up of traffic. Referring to the development of the cities, the initially fencing-off, centralising or functional-focussing function of traditional townscapes - around centrally located functional buildings such as churches, fortresses or town halls which were relevant for the collective - are forgotten as a result of the settlement growing beyond the city walls. The auto mobilisation of cities results in forgetting how to perceive them, which happened by way of experiencing them on foot. Connerton's argument sounds somewhat culturalpessimistic when he states that spatial memory requires a certain degree of stability and that this stability of the local system is destroyed by the production of speed and the use of mobility machines. Experiencing space through the windshield is something like watching a film. After all, Connerton's considerations on oblivion are influenced by Benjamin's arguments - always, it is

references as well as the motif of the paradigm change as unfolded by Thomas S. Kuhn. Paradigms proliferate as long as, due to their complexity, a completely new paradigm can push through. Kuhn (1962) calls this a scientific revolution. Both aspects will be discussed in more detail later. 
about the loss of what can be immediately experienced, the haptic and thus the aura of the object.

If we leave away those aspects as looking cultural-critical and conservative, there remains the diagnosis that innovation in and the growth of societies result in changes in their culture. This creates the preconditions for traditional structures or generally for what is old falling into oblivion - such a simple statement does not require justification, after all. ${ }^{61}$

Thus, it comes hardly as a surprise that such ways of automatic oblivion are welcomed or are at least approved in modernisation-oriented contexts. In organisation and management research, sometimes the old is associated with organisational memory as an obstacle for open-mindedness towards innovation and organisational adjustment processes. However, there are also attempts to focus on the costs resulting from processes of automatic oblivion. One aspect that is considered a problem is oblivion due to a loss of human capital, such as experienced staff members leaving the company. ${ }^{62}$

To sum it up: we may state that these kinds of oblivion - filed under "natural" or "automatic" - may also be applied intentionally or instrumentally right when being identified. The list is incomplete; however, it demonstrates that the disappearance of knowledge accompanies any access to knowledge. The idea that each individual act of not making use also rules out any further access is not helpful in this context. In certain fields of knowledge, oblivion rather looks like things slowly sinking or being wiped out. How swiftly this sinking down happens depends on connecting this knowledge to the interpretations and actions required by each respective context or situation. The process may happen sooner here or later there; in any case, it goes on unnoticed, which suggests the conclusion that even oblivion will be forgotten.

\subsubsection{The "Culture" of Oblivion}

The following review is not supposed to be another cultural history of oblivion - which has already been presented by Harald Weinrich (2004), by the

61 In a similar way and also not without cultural-critical undertones, Konrad P. Liessmann reports on the card index system of the Austrian National Library being replaced by an electronic index system. "Where the new declares itself to be the new, the hold must disappear - and this means at once. Otherwise the new would not be new but only an alternative possibility" (Liessmann, 2000, p. 8).

62 Organisational oblivion is on the one hand understood to happen automatically, on the other hand as happening intentionally-volitionally in the context of the theory of organisational learning. Due to its closeness to the tradition of sociological-social-scientific theories, this topic will be taken up again elsewhere. For the start we may refer to the overview by James P. Walsh and Gerardo Rivera Ungson (1991), in which some problems of organisational memory are discussed. 
way. Instead, it is about extending the scope of meaning, grasping the mythical or historical roots of certain meaning dimensions of a kind of oblivion that has been applied purposefully and has become an element of culture. This allows for working out the foundation for interpreting the various oblivion problems against a social-theoretical background.

At first sight, it becomes evident that oblivion was not only discussed in the context of a specific historical age - as a problem of antiquity, the Middle Ages or the modern age. Accordingly, a high number of hints are to be found since Greek antiquity. After a short overview of some early perspectives on oblivion, the review will be extended to the fields of historical science, literature, psychology and the neurosciences, and political sciences. However, the thus resulting manifoldness of different approaches allows for identifying other basic patterns of oblivion. Individual attempts at a classifying compilation have already been presented, except the excellent compilation of sometimes very disparate motifs - these are not satisfying, as they are just variants of cultural phenomena associated with cultural oblivion. ${ }^{63}$

The discussion of culture-specific kinds of oblivion starts with the exuberant memory performance of social groups, which appear as "nature" and require assessment. The motif has already been presented in terms of the oblivion command of Athenian democracy and the considerations by Nietzsche. The preceding cultivation of memory may well have happened through remembrance policy or forgetting remembrance or - and this is perhaps the most frequently described case - by announcing an "hour zero" or "leaving for fresh fields". In any case, the function of oblivion means becoming open-minded towards future options by relieving oneself from a burdening history. Those activities triggering or even enforcing oblivion can be attributed to individuals, social groups or collectives, in the context of which it would have to be decided if collective oblivion happens due to the tacit agreement of the many or due to the rulership of a few people.

The first group of culture-specific kinds of oblivion results from a tacit agreement among many. Reaching back to an established ethnologicalculture-anthropological concept is close at hand in this context. The taboo "institutionalises" a "critical situation" in the sense of levelled distances until an absolute distance has been achieved: "do not touch!" (Gehlen, 1986, p. 213). It controls attention and names things that must not be done. Taboos serve for distinguishing the sacred from the profane and consolidate power relations.

63 These are first of all the two typologies by Paul Connerton (2008) and Aleida Assmann (2012). 
By setting guidelines - as Arnold Gehlen states - in critical situations, taboos prevent the social order from being irritated.

One way of silencing is collectively not discussing shameful deeds of the past or past defeats. Remembering something that cannot be changed is perceived as a burden and, most of all, an obstacle to coping with current problems. ${ }^{64}$ The period after World War II is - most of all in Germany - connected to such silencing.

In the West German post-war society, communicative silencing allowed for a retreat from the political public; the immediate past disappeared from the awareness of society, discussing the past was left to the 'soakaway' of coping with it in private life. (Assmann, 2007, p. 179) ${ }^{65}$

Remarkable with this phenomenon is that it caused considerable outrage in the course of social-scientifically reflecting on Germany's post-war society. Nevertheless, it was identified as being culture-specific and thus supraindividual. Accordingly, Theodor W. Adorno does not accept the psychological mechanisms of suppression as the sole cause and identifies silencing as a strategic-instrumental nature - however less against the background of adjustment logics than rather against the background of maintaining traditional power structures.

The effacement of memory is more the achievement of an all to alert consciousness than its weakness when confronted with the superior strength of unconscious processes. In the forgetting of what has Gary scarcely transpired there resonates the fury of one who must first talk himself out of what everyone knows, before he can then talk others out of it as well. (Adorno, 1998, p. 92)

Frequently, oblivion by way of silencing proves to be deceitful - however, it may be connected to the hope of being redeemed from the memory of a bad past. ${ }^{66}$ By discretion, Hermann Lübbe recognizes a kind of communicative silencing (Lübbe, 1983). Such a kind of silencing is characterised by all participants consciously keeping a topic out of public communication. Thus, it is a superficial kind of oblivion, in the context of which it must generally be asked if it is a process of or preparing for oblivion or if there is a "telling" silence in

64 Being silent about the past comes along - at least when it comes to the German post-war era - with some present-related actionism as described by Helmut Schelsky (1975) in his study on the Skeptic Generation.

65 Aleida Assmann calls being silent a strategy of oblivion.

66 Accordingly, Connerton (2008) lists also being silent under types of oblivion, attesting each specific ways of being silent to victims and to perpetrators. 
certain situations. In this context, Helmut König defines silencing, in contrast to being silent, as purposeful action:

Thus, the loss of memory is actually a refusal to communicate, at least if communication is tied to language. But speaking of communicative silencing maintains that this kind of excommunication is at the same time a way of communicating. (König, 2008, p. 525)

If, as König assumes, this silencing is based on purposeful behaviour or if it happens automatically by tacit agreement, it is difficult to say. ${ }^{67}$ True - tabooing may also be caused by a power relation, but this aspect shall be discussed in more detail below.

Another way is the victims being silent towards the perpetrators. It is considered an expression of ongoing powerlessness and may be counted among oblivion, at least on the surface. At first, it is obvious to suspect communicative silencing also there. Such a kind of communicative silencing serves for avoiding sanctions while at the same time tacitly keeping memories alive, which is something like pseudo-oblivion. However, such a discussion taboo need not necessarily be enforced by rulers. ${ }^{68}$ It may as well result from a diffuse mixture of individually or collectively perceived shame, uncertainty and insult. ${ }^{69}$

67 In view of Germany's NS-past, so far, the motif of psychoanalytical suppression has frequently been transferred to the collective - however e. g. Alexander and Margarethe Mitscherlich (1975, p. 28) understand the "collective denial of the past" to be a massphenomenon which has its origins with the subject, after all. In this concern, this strand of social psychology rests on a connection between individual and collective in the sense of Freud's mass psychology (see Freud, 1975; Mitscherlich, 1975). Just the same, however, we may assume that collective silencing comes from values-based discretion or, in other words, from a way of being silent which is, in a diffuse way, mutually anticipated. Then the taboo would rather have to be understood as having "grown" and not as having been "created".

68 Being forced to be silent, as it is practiced by the Italian Mafia under the name of "omertà", serves for preventing persecution. Oblivion is normatively enforced - by the state - by way of preventing communication. That under the surface this does not mean real oblivion becomes obvious from the necessity to enforce this practice rigidly, usually be way of murder (see Bestler, 2003). Aleida Assmann (2012, pp. 33-37) further differentiates the oblivion type of being silent as complicity by pointing out to the connection between the perpetrators being silent for the sake of their own defence, the symptomatic way of being silent of the victims, and society being silent as complicity.

69 The victims being silent is frequently discussed in the context of (sexual) abuse and violence and connected to suppression on the one hand, however to shame and thus duplicating the crime due to public attention on the other. The victims fear to suffer even more from accusing their tormentors. They must expect to live through what happened in the past once more while at the same time being stigmatised as victims. Nevertheless, the victims e. g. of mass rape being silent has a social background because of this inexpressibility, 
Ideas of relieving oblivion, of a tabula rasa or annulment sometimes result from the problem of being confronted with an abundance of information. The introduction of writing was such a relief, to begin with, as it relieves the necessity of keeping certain things "in mind". Apart from the necessity of being relieved of a "too much of information", the art of oblivion (ars oblivionalis) is as old as the art of memorising, the former coming from the desire to be no longer in need of remembering. Indeed, the success of the art of oblivion is frequently doubted; however, perhaps due to the spread of the science of history - from the mid-19th century on and in the context of the demand for comprehensive oblivion, it gains renewed popularity. In the following, we will shortly discuss the two motifs of this relieving kind of oblivion.

The insight that the introduction of writing may suspend the storing of knowledge in the mind is already found in Plato. By writing, whose benefit is storing knowledge that is not necessary for everyday life, he recognized a tool for oblivion. ${ }^{70}$ The principle of the systematic storing of knowledge as a symbol may also be transferred to archives, the archive organisation, the catalogue, being symbolically organised, and the archived objects being put out of the way when it comes to coping with everyday life. While connecting to Friedrich Georg Jünger, Aleida Assmann calls this kind of oblivion by archiving custody oblivion. ${ }^{71}$

Then, in the context of digital ways of archiving, the problem becomes evident that already information gained with the help of search engines by far overtaxes our perception and processing capacities: Manfred Osten points out a fundamental change of the memory function: "The memory, as yet well trained for dealing with self-generated associations and insights into connections, suddenly finds itself to be a habitualized user of storage capacities with technologically determined formal operations and being dependent on digital 'search engines'” (Osten, 2004, p. 78).

as it is not only about the subject having been violated but most of all also about the risks connected to the fight for recognition under the given social conditions.

70 In Phaedrus, Plato has Sokrates say: "For this invention will produce forgctfulness in the minds of those who learn to use it, because they will not practise their memory. Their trust in writing, produced by external characters which are not part of themselves will discourage the use of their own memory within them." (see also Frances A. Yates, 1999, p. 38 ).

71 See Aleida Assmann (2012) as well as Friedrich Georg Jünger (1957). A dystopia which is in line with this phenomenon was presented by Jules Verne who, in his story Paris au XX $X^{e}$ siècle, sketches the image of a technocratic world being hostile towards art, where poetry and fiction are stored at a library whose ordering procedures are highly bureaucratic and lengthy. There, as stated by Dietmar Rieger (2004, p. 21), the library is both a place of storing and a "cemetery". 
Indeed, search engines themselves already offer their solution for the surplus of information. Elena Esposito describes Internet search engines as

data-processing machines producing information as their product - and indeed not because they remember information but because based on the user's commands, which in each individual case generate a unique search path and thus a new memory structure, they newly constitute it each time. (Esposito, 2002, p. 357)

In line with the satisficing concept, ${ }^{72}$ according to which a too complex search for information stops with the first satisfying result, they control the organisation of search results by way of algorithms. ${ }^{73}$ The rules according to which each search request is dealt with and how this is done is a strictly kept company secret.

Relief by way of writing and archive is two-faced. On the one hand, it must be understood to be a relief through which complexity is reduced. On the other hand, however, these achievements are connected to a reflexive effect, for their realisation makes certain qualities and liberties disappear.

In philosophy, memory, remembrance and, thus connected, oblivion has frequently been discussed since the beginnings of written records. Often the starting point is preventing oblivion with the help of an ars memoriae. Remarkably, in this context, the worthwhile capability of keeping as much as possible in mind triggers a feeling of being burdened by an all too exact memory at the same time. When antiquity singer, poet, and memory artist Simonides of Keos offered the general Themistokles to teach him the art of memorising, the latter put him off by saying that he was rather interested in being taught the ars oblivionalis. His memory, he said, was already in a painfully good state so that he would feel well with being able to forget things once in a while. ${ }^{74}$ Thus, the art of oblivion is understood to be a relief of a surplus of knowledge that has resulted either from an exceptional talent, long-standing practice or

72 See Herbert A. Simon (1956). Even satisficing as a strategy of problem solving is a kind of oblivion.

73 On this see the elaborations by Wolfang Hagen (2011).

74 Among others, Cicero reminds to Simonides as the award-winning inventor of the art of memory - at about the year 264 BC Simonides of Keos had been honoured for having developed a system of memory aids. The source, a stone tablet, also mentions the invention of the flute or the introduction of grain (Yates, 1999). However, another story is also told. According to this, given the disaster of a collapsed hall, Simonides as the only survivor helped identifying the disfigured dead - before leaving the building he had memorised each seating place. Hans Haverkamp (1993) comments on this story as the birth of the art of memory from disaster which is understood as an oblivion event. The anecdote on the art of oblivion is told by Harald Weinrich (2004). 
optimisation of memory. Thus, throughout the history of philosophising on oblivion, we find the idea of informational overtaxing resulting from too much remembrance or knowledge.

Also, the idea of reliving oblivion given a surplus of memory has its roots in Greek mythology. For example, in Virgil's Aeneas legend, we find different motifs of remembrance and oblivion. After the Trojan War, the Trojan hero Aeneas leaves his home and sails to Africa, then to Italy. The story may be interpreted as a flight from the memory of the traumatic experience of fighting, being wounded and death. The journey is read as an allegory of an overwhelming memory fading all too slowly, of a "memory attack" coming along at first with paralysis and slowly developing prospects of a new future. Here, oblivion appears as being successively relieved of remembrance, which also happens in the form of "attacks" and, most of all, blocks the capacity to act. ${ }^{75}$

In his Untimely Considerations, 30 years old Friedrich Nietzsche discusses, under the title "On the Uses and Disadvantages of History for Life", indeed polemically, the question of how useful historical research is at all. To him, the science of history is the ideal type of the propensity of remembering the past and thus preventing it from falling into oblivion. To him, the problem is that too much remembrance is an obstacle to practical life. Nietzsche presents a typology of historicising interest consisting of three kinds: the monumental historian grasps the outstanding achievements of humanity to learn from them and teach and advise other people. The flip side of such a kind of memory is that only certain events are selected, whose exaggeration makes the "courageous to foolhardiness and the inspired to fanaticism" (Nietzsche, 1997, p. 71). The antiquarian historian keeps and adores the past - and this mostly indiscriminately. Everything past is worth being remembered. Thus connected is the danger that the antiquarian builds his "nest" in the past and either no longer perceives the present or does not know how to appreciate it - then scholarly routine circles around itself. Finally, in the context of critical history, the past is remembered to be broken and dissolved. According to Mephistopheles's words in Goethe's Faust, this kind of historicism characterises itself by the motto: "And rightly so: Since everything created, in turn deserves to be annihilated." Sometimes, says Nietzsche, life demands things to be forgotten. However, then the danger is that the critical historian sets himself or herself the judge of the past, even though he or she comes from a past he or she could not choose.

75 On this see the elaborations by Reinhart Herzog (1993); the motif of the surplus of information was also included by Connerton (2008), under the name of "annulment", for his typology of oblivion. 
Nietzsche's focus is on the antiquarian type, the person hostile towards education as the godfather of modern man. He describes him or her as being ill and weak due to historical education. Accordingly, Nietzsche says, occidental and in particular German modernity is characterised by a (lack of) culture obsessed with successorship which, given a too much of remembering the great past, wastes its time with archiving knowledge of the past, thus not only forgetting creative life but moreover consciously suppressing it. Nietzsche says that educating the youth consists of educating not free, educated minds but scholars - by communicating historical knowledge. Thus, when it comes to the most important aspects of youth, too much history is capable of "deracinating":

We know, indeed, what history can do when it gains a certain ascendancy, we know it only too well: it can cut off the strongest instincts of youth, its fire, defiance, unselfishness and love, at the roots, damp down the heat of its sense of justice, suppress our regress its desire to mature slowly with the counter-desire to be ready, useful, fruitful as quickly as possible, cast morbid doubt on its honesty and boldness of feeling; indeed, it can even deprive youth of its fairest privilege, of its power to implant in itself the belief in a great idea and then let it grow to an even greater one. (Nietzsche, 1997, p. 115)

Nietzsche's critique of history as a de-educating institution, by which man is characterised, addresses both the individual and the collective. He implies a concept of memory that is limited to a (pseudo-)cultural-educational memory. However, even in the everyday lives of individuals, this cultivated historical memory is powerful enough to affect both their attitude and their opportunities to shape their lives actively. With the help of the metaphors of ill/healthy on the one hand, he propagates a well-measured amount of past which is worth preserving and passing on - with a certain degree of sympathy for the great however that may be defined - while on the other hand speaking in support of comprehensive oblivion.

According to Weinrich's interpretation, no clear statement on remembrance or oblivion can be identified in Nietzsche. Whereas in earlier works, he still propagated the relieving function of oblivion, he later discusses the question of when things are inextinguishably kept in mind. His mnemotechnic, which he explains in "Genealogy of Morals", may be reduced to the sentence "One burns something in, so that it remains in the memory: only what never ceases to hurt remains in the memory" (Nietzsche qtd. in Weinrich, 2004, p. 131). Weinrich concludes that thus obviously, we have to do with two Nietzsches, one of them demanding art of oblivion, and the other one revoking it partly.

For a sociological interpretation of these fundamental insights on memory, remembrance and oblivion, we may state that Nietzsche does not present any 
analysis of (social) memory functions. Instead, he unfolds a diagnosis of his time which rests on a particular (de-)cultivation of memory while at the same time speaking out in support of cultivated - and that is organised and thus intentional or conscious in whichever ways - oblivion. He criticizes the historical sleeplessness of modernity which is an obstacle for salutary oblivion. In this sense, we may refer to Hans-Georg Gadamer here, who comments as follows on the kind of oblivion to be found in Nietzsche: ${ }^{76}$

In a way that has long been insufficiently noticed, forgetting is closely related to keeping in mind and remembering; forgetting is not merely an absence and a lack but, as Nietzsche in particular pointed out, the condition of the life of mind. Only by forgetting does the mind have the possibility of total renewal, the capacity to see everything with fresh eyes, so that what is long familiar fuses with the new into many leveled unity. (Gadamer, 1979, p. 15)

However, in times of digital archiving and impenetrable archives being impenetrably controlled by logarithms, there is sometimes the call for comprehensive oblivion. The myth of the millennium bug, a programme error that would put every computer to a halt at the turn of the millennium, was not only a scenario of fear but just as well a redemption fiction. Accordingly, in 1999 Hugo Loetscher suggested relieving oblivion concerning all digital data in the tremendous deleting party. ${ }^{77}$

Thus, the concept of relieving oblivion has two sides. On the one hand, it is about being relieved from painful remembrance as an obstacle to coping with life in the presence. On the other hand, an exaggerated orientation towards the past is addressed - we might call it nostalgia - whose effects on coping with the present are similarly dysfunctional, which is why a return to oblivion as a selection function and relief is recommended.

Some of the concepts presented so far represent ideas of conscious and intended - that is, volitional - oblivion. In a short essay titled "An Ars Oblivionalis? - Forget it!" philosopher Umberto Eco asks if intended oblivion is possible at all. ${ }^{78}$ For his critique of oblivion, he assumes that the art of oblivion would have to be the opposite of the art of memory. The art of memory is a semiotic practice making the absent present with the help of signs. The use of signs is - different in each case - accompanied by the "inner eye" becoming

76 This quotation is also used by Heinz Dieter Kittsteiner (1996) who, other than Weinrich, does not identify any ambivalent attitude with Nietzsche but deconstructs the latter's oblivion recommendation and assesses it for being contemporary.

77 Manfred Osten (2004, p. 83) in his critique of digitalisation adopts this suggestion by Hugo Loetscher.

78 See Umberto Eco (1988) as well as the summarising comment by Sybille Krämer (2000). 
aware of what is represented by the sign. However, now, asks Eco, are we supposed to use a sign to forget what it stands for? Oblivion, he says, is a "natural" phenomenon, whereas remembrance is a cultural technique. This argument is logical and explains why it is practically so difficult to forget something, such as an unhappy love purposefully. Nevertheless, it seems as if there is an art of oblivion using "natural" oblivion by purposeful, selective remembrance, pushing certain experiences out of what is relevant. This is possible both for the subject, by consciously providing for distraction, and in social relations, if external hints at memories are taken away or if memory communications are banned.

After the agreement of the many to be silent has been presented as at least temporary oblivion, and after both the potentials and the limits of desired oblivion in the social context have been presented, culture-specific kinds of oblivion which are explicitly established by a political will within groups remain. Also, in this concern, the documentation reaches as far back as the practices of conflict solving in Greek antiquity. Whereas the philosophical theory of memory deals first with ways of thinking while considering an idea of time, this now is most of all about the political-philosophical question of how far peaceful living together - particularly after conflicts - depends on mechanisms or imperatives of oblivion.

An early source of such a politically motivated kind of oblivion refers to Athens in the fourth century BC. The dictatorship of the Thirty Tyrants had been ended by the victory of an army of expatriates: the period of persecution and expulsion had resulted in a deep rift among the city's population and had unhinged their relationships. A new beginning seemed to be necessary, based on an oath of amnesty: the citizens were supposed not to bear each other any ill will - exclusively, the tyrants were to be held accountable and to be persecuted, but not their supporters. It is remarkable that, despite the massive injustice they had suffered, the victorious democrats even gave up on the possibility of legitimate retribution and made themselves subject to a ban on remembering the past. This oblivion imperative required forgetting both victory and anger and the desire for retribution - an enterprise to which not every involved party did contribute to the same degree. Subsequently, frequent executions happened in the course of which those being obstinate, who would not stop calling for retribution, lost their lives. ${ }^{79}$

Also, we find the development of oblivion imperative in the New Testament. Weinrich interprets the story of the adulterous woman (John 8) and the

79 On this see e. g. Nicole Loraux (1996), Christian Meier (2010) and also the second type of oblivion in Paul Connerton (2008). 
command "Go. From now on, sin no more." not only as an immediate connection of forgiving and oblivion. Rather, there he also identifies the beginning of the Christian practice of confession and atonement. God may forgive the Christian all sins if he or she is ready for atonement (Weinrich, 2004, pp. 165166). In the Christian faith, there is the possibility of a new beginning at any time, which finds expression not only by God's amnesty but most of all by telling the community of believers: not only the sinner is supposed to change his or her ways; also his or her social environment is told to make this possible.

In the philosophy of the 2oth century, by French existentialism there develops an interpretation of the idea of the possibility of a completely new beginning which focusses very much on the subject. Accordingly, homo existentialis drafts his/her identity exclusively given future actions. According to Jean-Paul Sartre, what is to be ignored is what the others think. Weinrich interprets this existentialist moral, which calls for forgetting what has happened and for drafting oneself only out of one's existential actions, against the background of post-war Europe with its collective "hour zero" amnesia or the "leaving for fresh fields" discourse. In contrast to this, it must be doubted that it is possible to hedge in the memory as the driving force of and criterion for action. ${ }^{80}$ After all, history demonstrates that already the next generation, that of the year 1968, had a strong interest in lifting this amnesia resulting from collective silencing (Weinrich, 2004, pp. 164-165).

Against the background of guilt and retribution in social relations, an agreement on oblivion appears as a way out of the threatening spiral of violence. The examples given here demonstrate that amnesia and amnesty may happen on the level of collectives, groups, or constitutions of social subjects. In this context, the focus is always on the question of coping with a "bad past" 81 and about the preconditions for a new beginning against the background of the insight that remembrance may be an obstacle for reconciliation and forgiveness. ${ }^{82}$

In this context, Paul Ricoeur distinguishes two kinds of oblivion: deep oblivion and visible oblivion. In the field of deep oblivion, relentless oblivion, which irretrievably deletes the traces of experience, and forgetting the immemorial contrast each other. Immemorial is what has become sedimented in the stock of knowledge and is thus not available for the reflective-remembering grasp of the individual. The variant of visible oblivion concerns everything which

8o However, there are indications that such suppressions might be necessary - Freud will be discussed elsewhere - in post-conflict societies. On the situation in post-war Germany see e. g. Hermann Lübbe (1983).

81 This refers to a book title by Christian Meier (2010).

82 See Avishai Margalit (1997) - oblivion effects resulting from truth and reconciliation commissions are discussed by Christoph Lau (2012). 
might be recollected. Among this there count both the psychoanalytic concept of suppression and escapist oblivion as a strategy of avoidance as well as, finally, selective oblivion, which happens automatically at the moment of any narration of the context of life: one is incapable of remembering everything and thus incapable of including everything into a biographic narration.

Transferred to organisations, the manifestations of deep oblivion may help with understanding organisational change. The analysis of visible oblivion, on the other hand, may uncover both experiences and taboos which cannot be narrated, and - when considering selective oblivion - it may point out inconsistencies of the great narrations of organisations. ${ }^{83}$ Already Ricoeur's considerations provide us with a differentiated classification of different kinds of oblivion which may also be generalised and transferred to other fields. The background of his oblivion theory, however, is conflict and the thus resulting trauma. Accordingly, his analysis does not result in the phenomenology of oblivion; instead, it amounts to a philosophical discussion of the question of how forgiveness and thus a new beginning might be possible.

Another kind of intentional oblivion is legitimately forgetting about a criminal act. Amnesty is not only a kind of reprieval or a way of remitting a penalty. It represents some "being-supposed-to-not-remember" (Simon, 1997, p. 29) at the level of society. ${ }^{84}$ Thus connected is the prospect of not being persecuted, however then rehabilitation in the sense of being accepted by society again. Great amnesty programmes are carried out, for example, in post-conflict societies, which are supposed to be enabled beginning anew. As organised oblivion, amnesty contributes to a process of reprocessing - each according to culturalpolitical specifics. Like in the example of the 30 Tyrants in ancient Athens, persecution happens only symbolically and aims exclusively at the "heads" of the old system - the bulk of "minor" perpetrators are granted amnesty.

Political scientist Gesine Schwan points out that such amnesties are the price to be paid for social peace by decoupling law and morals. Thus, the idea of "leaving for fresh fields" is risky, particularly for democratic structures (Schwan, 1997). In the present, amnesties are frequently connected to establishing so-called truth or reconciliation commissions which are also meant to give the victims recognition and a say.

83 A comprehensive analysis of the possibilities of forgiveness by way of oblivion is carried out by Paul Ricœur (2004b). An outline of the phenomenological classification of oblivion is to be found elsewhere (see Ricoer, 2994a) - a short overview is provided by Oliver Dimbath (2014).

84 In more detail Christian Meier (2010). 
Even if oblivion is supposed to be cleaned from any negative undertones, the concept of oblivion policy is rather a concept that seems suitable for uncovering political mischiefs - a combat term. If rulers issue a decree of oblivion, this raises the suspicion that an authoritarian system is fighting for survival (Connerton, 2008; König, 2010). Too obviously, any oblivion imperative looks like a hopeless enterprise connected to a high degree of suppression, which is much more likely to keep undesired memories alive in the underground. However, it has already been pointed out that, on the one hand, an oblivion decree may serve to bring peace and make it last. Philosopher Ernest Renan even assumes that any national identity must result from massive oblivion processes (Renan, 1995). On the other hand, history demonstrates that oblivion imperatives need not necessarily be "top-down" but may as well reflect a collectively shared desire of a social group. ${ }^{85}$ Also, this is a culture of oblivion that may also be analysed for its way of functioning. Thus, how a process of oblivion might happen or how it could be described if oblivion is understood to be functional for social cohesion and social peace is outlined in the following.

One variety of purposeful oblivion coming from antiquity is the oblivion punishment (damnatio memoriae). In a period of swiftly following radical changes of power relations, nobody could be sure to be on the "right" side. Any overthrow was connected to the possibility of overnight falling from being an office bearer and dignitary to being a public enemy. Perhaps it was due to general instability that a loss of reputation, for whatever reason, was not only punished by killing the protagonist. Rather, it was common practice to completely extinguish even the memory of the concerned person as far as ever possible by removing all reference points to possible memories (Weinrich, 2004). On the one hand, this produced the result that the concerned person's family and servants were killed or exiled. On the other hand, one erased all other hints at the person's existence - inscriptions of names and sculptures. The new beginning, however it was supposed to happen, was supposed to start as being unburdened and total as ever possible. ${ }^{86}$

Sometimes, the damnatio memoriae is interpreted against the contrast between old and new or traditional and modern. For example, historian Jacques Le Goff, when discussing the difference between new and modern, states as follows: "New signifies more than a break with the past, a forgetting,

85 See Paul Connerton's (2008) seventh type.

86 See the compilation by historians Gerald Schwedler, Sebastian Scholz and Kai M. Sprenger (2012). 
an erasure, or an absence of the past." (Le Goff, 1992, p. 26). ${ }^{87}$ Moreover, this motif may be completed by quoting Konrad P. Liessmann:

The old cannot simply disappear. Of course: in less cultivated environments it is destroyed and survives only by the traces of its destruction. Given more delicate ways of life, however, it suffers the fate of being aestheticized. Having lost any function, now the old reveals its beauty which, as long as it was in use, could not be noticed. [...] It has lost its function, on the other hand it has gained a meaning. (Liessmann, 2000, p. 10)

If the old is bereaved of its function and functionally replaced by the new, it is no longer punished by purposeful oblivion. Nevertheless, the bulk of the old is destroyed, only individual items are preserved - for reasons of nostalgia or delight. Forgotten are the many levels of meaning it showed when still fulfilling its original functions.

One last kind of oblivion to be mentioned here also consists of an instrumental loss of knowledge. Also, in this case, the actions of an oblivion actor cause oblivion with others. At last, the modern capitalist consumer society depends on making the life cycles of products ever shorter. This applies to being fascinated by the new and the thus connected ephemerality. At the same time, also the good or product can, right from the beginning, be programmed for limited durability. Against this background, when household appliances stop working shortly after the warranty period has expired, this is not only a modern myth but may as well be intended.

In the context of his considerations on the topography of oblivion, Connerton, who refers to Friedrich Engels, points to the fact that e. g., the decay of working-class quarters was intended right from the beginning, to be able to rebuild forty years later. Connerton finds his assumption that urban change - and thus the urban topography falling into oblivion - may be interpreted against the background of building construction and civil engineering confirmed by a study of 1936 . There it is demonstrated that most buildings in London, as far as they were not subject to monument protection, were renovated thirty years later and demolished sixty years later - in the United States, he says, this life cycle is even shorter (Connerton, 2009, p. 117). In the context of intended obsolescence, the loss of knowledge refers to utilisation knowledge applied to the use of tools connected with a particular fetish character of the products. Through sales-strategically motivated innovation cycles, there happens a forced change of utilisation patterns and tools. This begins with fashion

87 See also the "Manifesto of Futurism" mentioned by Connerton (2008). 
and ends with innovations that increase the change value but not the utility value.

\section{$2.4 \quad$ The Ambivalence of Oblivion}

Oblivion, we may say after having sounded out its different kinds and (dis-) functions, is judged on ambivalently. On the one hand, it appears as a deficit and a threat to origin, continuity and identity. On the other hand, one does not only accept that it happens more or less "automatically" and can at best be controlled to a certain degree. It also becomes apparent that sometimes it seems to be highly desirable to be able to forget about certain things. The desire to forget refers to a bad past, the memory of which is perceived as a burden, and unnecessary knowledge that, if taken into consideration, distracts from allegedly relevant aspects of coping with current situations - always regarding a future to be shaped. In this context, it becomes evident that oblivion must be considered from different points of view. Among others, we must distinguish between subject relations, aiming at the more or less "natural" forgetfulness of the individual or collective consciousness, and object relations. Object relations refer to the social ways of dealing with objects which - even as persons may (be forced to) fall into oblivion.

The discussion of social time has demonstrated that knowledge must be understood according to the categories of past, present and future. Knowledge results from past experiences that - like experiences made by the consciousness - are kept available to define a current situation and plan future activities. Thus, oblivion, which becomes recognizable only by encountering traces, consists of the one hand of the consciousness's (or a consciousness system's) retrospective insight that it had knowledge in the past, whose reconstruction is not possible without effort (remembering). On the other hand, the process of oblivion may be reflected on, or we may consider the conditions under which keeping knowledge contents ready is useful or rather an obstacle under respectively different cultural conditions.

In the next section of this study, reaching back to sociological theories will shed light on the subject of such differentiated oblivion, which also refers to social reference frames. Doing so is meant to achieve a concept of social oblivion and see where the questions about the connections of time and action are already discussed in the context of sociological theoretical work. 